\title{
Polyamine Oxidase 5 loss-of-function mutations in Arabidopsis thaliana trigger metabolic and transcriptional reprogramming and promote salt stress tolerance.
}

Xavier Zarza ${ }^{1,2}$, Kostadin E. Atanasov ${ }^{1}$, Francisco Marco ${ }^{3}$, Vicent Arbona ${ }^{4}$, Pedro Carrasco ${ }^{5}$, Joachim Kopka $^{6}$, Vasileios Fotopoulos ${ }^{7}$, Teun Munnik ${ }^{2}$, Aurelio Gómez-Cadenas ${ }^{4}$, Antonio F. Tiburcio ${ }^{1}$ and Rubén Alcázar ${ }^{*}$

${ }^{1}$ Department of Natural Products, Plant Biology and Soil Science. Laboratory of Plant Physiology. Faculty of Pharmacy. University of Barcelona. Barcelona, Spain

${ }^{2}$ Department of Plant Physiology, Swammerdam Institute for Life Sciences, University of Amsterdam, Amsterdam, The Netherlands

${ }^{3}$ Departamento de Biología Vegetal, Universidad de Valencia, Facultad de Farmacia, Burjassot, Spain.

${ }^{4}$ Departamento de Ciencias Agrarias y del Medio Natural, Universitat Jaume I, Campus Riu Sec, E- 12071 Castelló de la Plana, Spain.

${ }^{5}$ Departamento de Bioquímica y Biología Molecular, Universidad de Valencia. Facultad de Ciencias Biológicas, Burjassot, Spain

${ }^{6}$ Max Planck Institute for Molecular Plant Physiology, Golm, Germany

${ }^{7}$ Department of Agricultural Sciences, Biotechnology and Food Science, Cyprus University of Technology, P.O. Box 50329 Limassol, Cyprus

*Author for correspondence: Rubén Alcázar, Faculty of Pharmacy, University of Barcelona, Avda. Joan XXIII 27-31, 08028 Barcelona, Spain. Tel. +34 934024492; e-mail: ralcazar@ub.edu

RUNNING TITLE: A role for thermospermine in salt stress tolerance

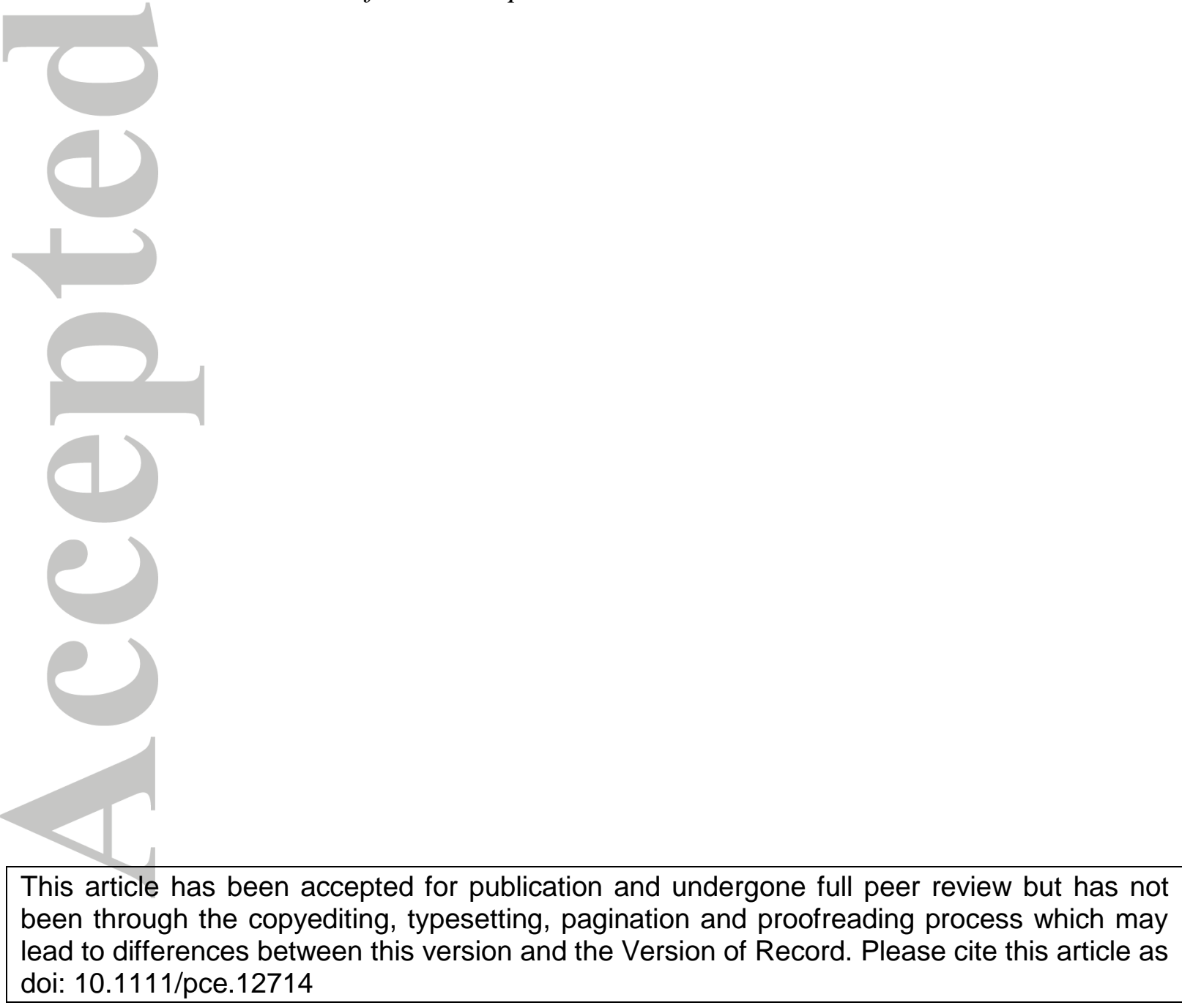

This article is protected by copyright. All rights reserved. 


\section{ABSTRACT}

The family of polyamine oxidases (PAO) in Arabidopsis (AtPAO1-5) mediates polyamine (PA) back-conversion, which reverses the PA biosynthetic pathway from spermine, and its structural isomer thermospermine (tSpm), into spermidine and then putrescine. Here, we have studied the involvement of PA back-conversion in Arabidopsis salinity tolerance. AtPAO5 is the Arabidopsis PAO gene member most transcriptionally induced by salt stress. Two independent loss-of-function mutants (atpao5-2 and atpao5-3) were found to exhibit constitutively higher tSpm levels, with associated increased salt tolerance. Using global transcriptional and metabolomic analyses, the underlying mechanisms were studied. Stimulation of abscisic acid and jasmonates (JA) biosynthesis, and accumulation of important compatible solutes, such as sugars, polyols and proline, as well as TCA cycle intermediates were observed in atpao5 mutants under salt stress. Expression analyses indicate that tSpm modulates the transcript levels of several target genes, including many involved in the biosynthesis and signaling of JA, some of which are already known to promote salinity tolerance. Transcriptional modulation by $\mathrm{tSpm}$ is isomer-dependent, thus demonstrating the specificity of this response. Overall, we conclude that tSpm triggers metabolic and transcriptional reprogramming that promotes salt stress tolerance in Arabidopsis.

Keywords: Polyamines, polyamine oxidase, thermospermine, salt tolerance, jasmonates, salt stress, metabolomics.

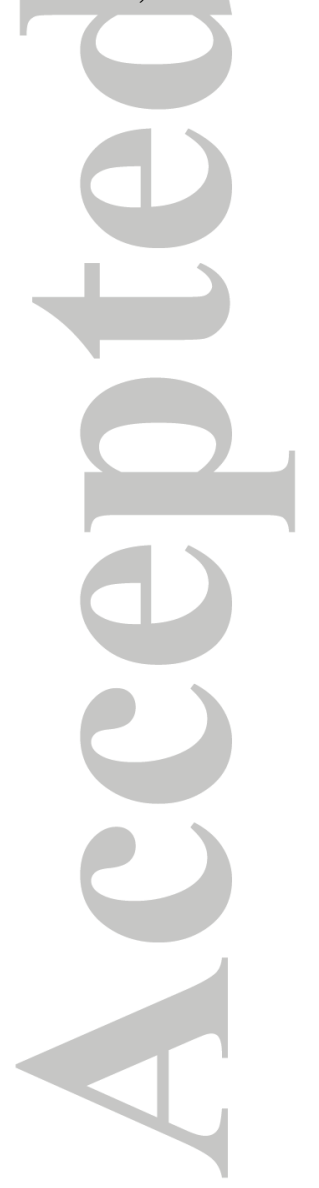




\section{Summary statement}

Arabidopsis atpao5 loss-of-function mutants exhibit constitutive accumulation of thermospermine (tSpm) that associates with enhanced salt tolerance. tSpm triggers transcriptional and metabolic changes that involve promotion of ABA and JA pathways, accumulation of TCA cycle intermediates, compatible solutes along with other effects that additively contribute to salt tolerance. We provide evidence for the involvement of tSpm in plant abiotic stress tolerance.

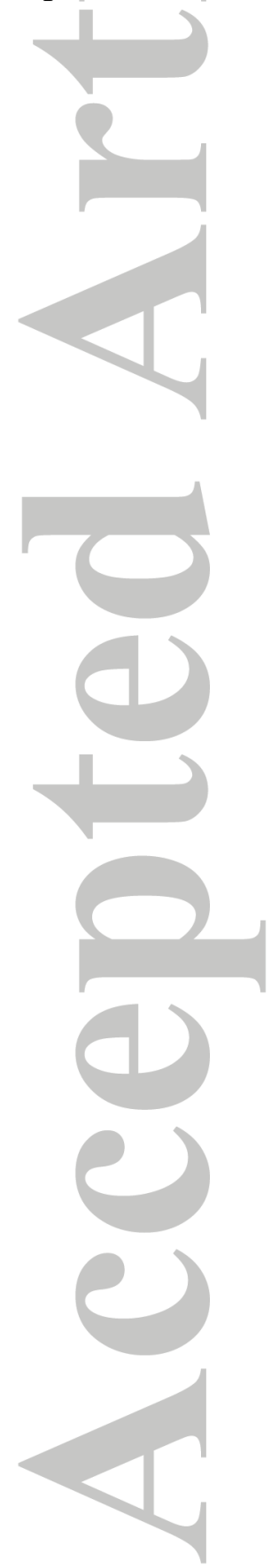




\section{INTRODUCTION}

Salinity affects more than 800 million hectares of arable land around the world and it has a detrimental impact on crop yield (Hasegawa 2013). To cope with saline stress, plants have evolved mechanisms that involve discrimination of ions taken up by roots, osmotic adjustment, sequestering of ions into separate cellular compartments, and tolerance against ionic toxicity (Munns \& Tester 2008). Polyamines (PAs) are organic amines the levels of which increase in response to various stress conditions, including salinity. Genetic approaches indicate that these compounds are required to cope with abiotic constrains, although their modes-of-action are not fully elucidated (Cona et al. 2006; Alcázar et al. 2006b; Kusano et al. 2008; Alcázar et al. 2010; Tiburcio et al. 2014). In the glycophyte species, Arabidopsis thaliana (hereafter referred to as Arabidopsis), the most abundant polyamines (PAs) are the diamine, putrescine (Put), the triamine, spermidine (Spd), and the tetraamines, spermine (Spm) and its structural isomer thermospermine (tSpm). Biosynthesis of PAs requires the presence of arginine or ornithine, from which Put is synthesized. Both amino acids are derived from glutamate, a derivative of the TCA cycle intermediate 2oxoglutarate, which is also precursor of the compatible solute proline (Alcázar et al. 2010). Spd is synthesized from Put via co-action of Spd synthase and S-adenosylmethionine decarboxylase (SAMDC). The latter is also involved in the biosynthesis of Spm and tSpm, by Spm synthase (SPMS) and tSpm synthase (ACAULIS5, ACL5), respectively. Despite their structural similarity, Spm and tSpm isomers exhibit strikingly different biological functions and their biosynthesis is differentially regulated (Takahashi \& Kakehi 2010; Vera-Sirera et al. 2010; Takano et al. 2012).

In Arabidopsis, salt stress up-regulates SPMS expression (Urano et al. 2003; Bagni et al. 2006; Tassoni et al. 2008; Naka et al. 2010). Long-term salt stress in this and other species leads to increased Spm levels (Naka et al. 2010; Alet et al. 2012). Genetic engineering of the PA pathway and correlational studies using cultivars with contrasting salinity tolerance, suggest that Spm accumulation is a metabolic hallmark often associated with enhanced salt tolerance (Yamaguchi et al. 2006). However, sensitivity to salinity of Arabidopsis mutants impaired in tSpm biosynthesis (acl5-1) or both tSpm and Spm (acl5-1 spms-1) is higher than that of Spm deficient (spms-1) or wild-type plants (Yamaguchi et al. 2006; Alet et al. 2012). Therefore, a potential contribution of tSpm to salt stress tolerance could not be ruled out. Evidence indicate that PA-triggered salinity tolerance is independent of the SOS (Salt Overly Sensitive) signaling pathway (Yamaguchi et al. 2006; Alet et al. 2012) and may involve 
calcium signaling (Yamaguchi et al. 2006), modulation of various cation and $\mathrm{K}^{+}$channels (Pottosin \& Shabala 2014), $\mathrm{H}^{+} /$ATPase activities (Pottosin et al. 2014), protein posttranslational modifications such as $S$-nitrosylation, carbonylation and tyrosine nitration (Tanou et al. 2014), enzymatic and non-enzymatic ROS detoxification (Saha et al. 2015), among others (Alcázar et al. 2010). However, the overall picture remains largely undefined and global transcriptional and metabolic approaches are, to our knowledge, not yet reported.

Free PA levels are not only determined by their biosynthesis but also by their oxidation (Cona et al. 2006). In recent years, several genes have been characterized that are involved in PA oxidation (Moschou et al. 2012; Tavladoraki et al. 2012). PA oxidation requires the activity of copper-containing amine oxidases (CuAOs) or FAD-containing polyamine oxidases (PAOs). Both types of amine oxidases exhibit different substrate preferences. While Put is preferentially oxidized by CuAOs, higher molecular weight PAs ( $\mathrm{Spd}, \mathrm{Spm}$ and tSpm) are PAO substrates (Cona et al. 2006). PAOs are classified into two main families, depending on whether they terminally oxidize PAs or catalyze the PA back-conversion, which reverses the PA biosynthetic pathway releasing $\mathrm{H}_{2} \mathrm{O}_{2}$ (Moschou et al. 2012; Tavladoraki et al. 2012). Arabidopsis carries five AtPAO genes (AtPAO1-5), all known to be involved in PA backconversion (Tavladoraki et al. 2006; Kamada-Nobusada et al. 2008; Moschou et al. 2008c; Takahashi et al. 2010; Fincato et al. 2011; Ahou et al. 2014; Kim et al. 2014). Among them, AtPAO5 encodes a cytosolic PAO the expression of which is restricted to vascular tissue, which is also the site of synthesis of tSpm by ACL5 (Kim et al. 2014). AtPAO5 preferentially catalyzes the conversion of tSpm to Spd, but not of Spd to Put (Ahou et al. 2014; Kim et al. 2014). Consistent with the proposed catalytic activity of AtPAO5, atpao5 mutants accumulate higher levels of tSpm under normal growth conditions (Ahou et al. 2014; Kim et al. 2014). Therefore, AtPAO5 and ACL5 activities can fine-tune the balance between tSpm and Spm.

PA mechanisms of action might involve cross-regulation with different hormones such as gibberellins (Alcázar et al. 2005), ethylene (Bitrián et al. 2012), auxins (Tong et al. 2014) or abscisic acid (ABA) (Alcázar et al. 2006a; Cuevas et al. 2008a). Recently, an apoplastic $\mathrm{CuAO}(\mathrm{AtAO})$ that is expressed early during root vascular tissue differentiation, has been shown to mediate jasmonic acid-triggered early protoxylem differentiation (Ghuge et al. 2015). In addition to the involvement of jasmonates (JA) in plant development, wounding and biotic-stress interactions, recent evidence indicate that JA contribute to salt stress 
tolerance (Kazan 2015). Overexpression of the JA biosynthesis gene ALLENE OXIDE CYCLASE 1 (AOC1) from wheat (Triticum aestivum) in Arabidopsis leads to higher JA levels, and this is associated with increased salt tolerance independent of ABA and dependent of MYC2 (Zhao et al. 2014b). Furthermore, overexpression of the wheat 12OXOPHYTODIENOATE REDUCTASE 1 (OPRl) in Arabidopsis promotes ABA dependentsalt tolerance and enhanced ROS detoxification (Dong et al. 2013). Hence, JA triggers both ABA-dependent and independent salinity tolerance in this species.

Overexpression of PA biosynthetic genes has been successfully applied for the manipulation of PA levels in plants (Alcázar et al., 2006). However, isolation of PA-catabolism mutants emerges as an alternative approach for the accumulation of PAs. It is expected that such approaches should help to further define PA-related biological functions. In this work, we have studied the involvement of PA back-conversion during salt stress in Arabidopsis. Expression analyses indicate that AtPAO5 is the Arabidopsis PAO gene family member most transcriptionally responsive to salinity. Phenotypic characterization of two atpao5 mutant alleles evidences that AtPAO5 loss-of-function leads to salinity tolerance. Through global transcriptional and metabolic analyses, we identify that salt tolerance in atpao5 associates with early boosting of ABA biosynthesis and higher JA levels, followed by the accumulation of important compatible solutes, TCA cycle intermediates, and transcriptional modulation of several target genes by tSpm. Collectively, the results indicate that tSpm has a protective role against salt stress in Arabidopsis.

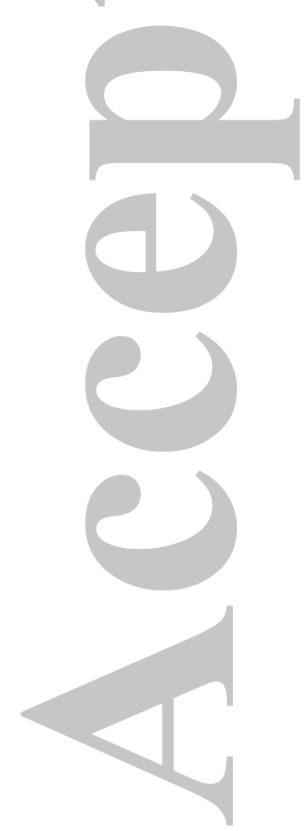




\section{MATERIALS AND METHODS}

\section{Plant material and growth conditions}

Arabidopsis thaliana wild-type (Col-0), atpao5-2 (N553110) and atpao5-3 (N509671) TDNA insertion mutants were obtained from the Nottingham Arabidopsis Stock Centre (University of Nottingham, Loughborough, UK). Seeds were sown on a mixture of soil and vermiculite (3:1) and stratified 4 days in the dark at $4{ }^{\circ} \mathrm{C}$ to synchronize germination. Plants were grown under $16 \mathrm{~h} / 8 \mathrm{~h}$ light/dark cycles, $100-125 \mu \mathrm{mol}$ photons $\mathrm{m}^{-2} \mathrm{~s}^{-1}$ of light intensity, $21 \pm 2{ }^{\circ} \mathrm{C}$ and $70 \pm 10 \%$ relative humidity. Salt treatments in adult plants were performed by irrigation of 4-week-old plants grown on soil with incremental concentrations (50 mM, 100 $\mathrm{mM}$ and $150 \mathrm{mM}$ ) of $\mathrm{NaCl}$ every 4 days (Gaxiola et al. 2001). Samples from rosette leaves were harvested at different time points, frozen in liquid nitrogen and stored at $-80{ }^{\circ} \mathrm{C}$ until further analysis. Salt treatment of Arabidopsis seedlings was performed as follows: different genotypes were germinated on a nylon mesh $(\varnothing 43 \mu \mathrm{m})$ placed on top of $0.5 \times$ MS-MES medium (Murashige \& Skoog, $0.5 \mathrm{mM}$ MES, $1 \%$ sucrose, $1 \%$ agar, pH 5.7 with $\mathrm{KOH}$ ) under sterile conditions. Ten days after germination, the nylon mesh with seedlings was transferred to the same medium supplemented with $50 \mathrm{mM} \mathrm{NaCl}$ for 3 days. Seedlings were then transferred to $0.5 \times$ MS-MES medium supplemented with $225 \mathrm{mM} \mathrm{NaCl}$. Chlorophyll quantification was determined in the different genotypes five days after $225 \mathrm{mM} \mathrm{NaCl}$ treatment.

\section{Identification of atpao5-2 and atpao5-3 mutants}

Confirmation of the identity of the mutants, and isolation of homozygous lines, was performed by PCR amplification and sequencing using a combination of gene- and T-DNAspecific (SALK-LB) primers (Table S1). Briefly, genomic DNA from individual plants was extracted using DNeasy Plant Minikit (Qiagen) and used as template for PCR amplification with ExTaq DNA polymerase (Takara). Amplified bands from SALK-LB and gene-specific combinations were separated in $1 \%(\mathrm{w} / \mathrm{v})$ agarose gels stained with $\mathrm{EtBr}$ and purified by gel scission using Qiaquick Gel Extraction Kit (Qiagen). Purified DNA was used for sequencing using the same primer set. The position of T-DNA insertion was confirmed by local alignment with the AtPAO5 gene (www.ebi.ac.uk/Tools/msa/muscle) and BLAST query (www.arabidopsis.org). Transcript levels of AtPAO5 in the homozygous lines were determined by RT-PCR from cDNA of atpao5-2 and atpao5-3 mutants using the AtPAO5specific primers and the following PCR conditions: $95^{\circ} \mathrm{C} 5 \mathrm{~min}, 30$ cycles $\left(95^{\circ} \mathrm{C} 15 \mathrm{sec}, 55\right.$ 
$\left.{ }^{\circ} \mathrm{C} 20 \mathrm{sec}, 72{ }^{\circ} \mathrm{C} 1 \mathrm{~min}\right), 72{ }^{\circ} \mathrm{C} 10 \mathrm{~min}$; and primers specified in Table S1. Single T-DNA insertion lines were confirmed by the occurrence of 1:3 segregation for the presence of kanamycin resistance gene (NTPII) in the progeny of segregating atpao5-2 and atpao5-3 mutants.

\section{Determination of PA levels}

The levels of free Put, Spd and Spm were determined by high-performance liquid chromatography (HPLC) separation of dansyl chloride-derivatized PAs as described (Alcázar et al. 2005). Free tSpm levels were determined by PA benzoylation according to Kim et al. 2014.

\section{Determination of water content}

Water content (WC) was determined using the fresh weight (FW) and dry weight (DW) and calculated as \% WC $=(\mathrm{FW}-\mathrm{DW}) / \mathrm{FW}$. Dry weight was obtained after drying samples at 60 ${ }^{\circ} \mathrm{C}$ for $48 \mathrm{~h}$. The shoots of at least ten individual plants were used to calculate $\%$ WC in every point of analysis and genotype.

\section{Determination of chlorophyll levels}

Chlorophyll extracts were obtained from leaves of plants grown on soil or seedlings grown in vitro at indicated time-points, using buffered acetone (acetone/Tris-HCl buffer 80:20 vol pH 7.8). Total chlorophyll levels were calculated according to Porra (2002).

\section{Determination of sodium levels}

For the determination of $\mathrm{Na}^{+}$content, lyophilized shoot tissue was digested in concentrated nitric acid at $80^{\circ} \mathrm{C}$ for $1 \mathrm{~h}$. After digestion, the acid was diluted with double deionized water. Mineral element concentrations in the supernatants were determined by ICP-OES (Inductively Coupled Plasma Optical Emission Spectroscopy; Perkin Elmer Optima 2000).

\section{Determination of ABA and JA levels}

ABA and JA levels were analyzed by HPLC coupled to Tandem Mass Spectrometry (Durgbanshi et al. 2005). Briefly, the plant tissue was extracted in ultrapure water using a tissue homogenizer. Before extraction, samples were spiked with $100 \mathrm{ng}$ of $d_{6}$-ABA and dhJA (Gómez-Cadenas et al. 2002; Durgbanshi et al. 2005). After extraction and centrifugation, the $\mathrm{pH}$ of the supernatant was adjusted to 3.0 and partitioned twice against diethyl ether. The 
organic layers were combined and evaporated. The dry residue was resuspended in a solution of water: methanol (9:1, v/v), filtered and injected into a HPLC system (Alliance 2695, Waters Corp.). The phytohormone fraction was separated on a reversed-phase C18 column using methanol and $0.01 \%$ acetic acid in water as solvents. The mass spectrometer, a triple quadrupole (Quattro LC, Micromass Ltd.), was operated in negative ionization electrospray mode and the different plant hormones were detected according to their specific transitions using a multi-residue mass spectrometric method as described in detail by Durgbanshi et al. 2005.

\section{Determination of hydrogen peroxide levels}

Hydrogen peroxide levels were determined as described by Loreto \& Velikova (2001). Frozen leaf material $(\sim 0.1 \mathrm{~g})$ was homogenized on ice with $0.1 \%(\mathrm{w} / \mathrm{v})$ trichloroacetic acid. The homogenate was centrifuged at $15,000 \mathrm{~g}$ for $15 \mathrm{~min}$ at $4{ }^{\circ} \mathrm{C}$. Then, $0.5 \mathrm{ml}$ of the supernatant was mixed with $0.5 \mathrm{ml}$ of $10 \mathrm{mM}$ potassium phosphate buffer $(\mathrm{pH} 7.0)$ and $1 \mathrm{ml}$ of $1 \mathrm{M} \mathrm{KI}$. The absorbance of the assay mixture was determined at $390 \mathrm{~nm}$ and the content of $\mathrm{H}_{2} \mathrm{O}_{2}$ calculated based on standard curves of known hydrogen peroxide concentrations.

\section{Microarray expression analyses}

Four-week-old atpao5-3 and wild-type plants were used for microarray analyses. RNA samples were extracted in triplicate from pooled samples of five plants per genotype using TRIzol reagent (Invitrogen). Transcriptomes were analyzed using $1 \mu \mathrm{g}$ of total RNA as starting material. Targets were prepared with MessageAmp II-Biotin Enhanced Single Round aRNA Amplification Kit (Ambion) and hybridized to ATH1 gene chips (Affymetrix). Microarray data and gene ontology (GO) classification were analyzed using GeneSpring GX 10 software (Agilent Technologies) and DAVID (Database for Annotation, Visualization and Integrated Discovery) v6.7 (Huang et al. 2009). A threshold of significance at $P=0.05$ and a cutoff value of $\geq \pm$ two-fold change was used for the identification of genes that were differentially expressed. Microarray data has been deposited in ArrayExpress under accession number E-MTAB-3817.

\section{Quantitative real time PCR gene expression analyses}

Total RNA isolated from 4-week-old plants or 7-days-old seedlings, as indicated, was extracted using TRIzol reagent (Invitrogen). Two micrograms of RNA was treated with DNAse I (Invitrogen) and first strand cDNA synthesized using Superscript II (Invitrogen) and 
oligo dT. Quantitative real-time PCR using SYBR Green I dye method was performed on Roche LightCycler 480 II detector system following the PCR conditions: $95^{\circ} \mathrm{C} 2 \mathrm{~min}, 40$ cycles $\left(95^{\circ} \mathrm{C}, 15 \mathrm{~s} ; 60^{\circ} \mathrm{C}, 30 \mathrm{~s} ; 68^{\circ} \mathrm{C}, 20 \mathrm{~s}\right)$. Standard curves were performed for quantification. Primers used for gene expression analyses are listed in Table S1. qRT-PCR analyses were always performed on three biological replicates with three technical replicates each using ACTIN2 (AT3G18780) as housekeeping gene.

\section{GC/MS metabolite profiling}

Metabolite profiling was performed from leaf samples $(120 \mathrm{mg})$ of wild-type and atpao5-3 plants using seven independent biological replicates for each time-point under study. Samples were shock frozen in liquid nitrogen and the fraction enriched in polar primary metabolites was prepared and processed as described (Erban et al. 2007). Gas chromatography coupled to electron impact ionization-time of flight-mass spectrometry (GC/EI-TOF-MS) was performed using an Agilent 6890N24 gas chromatograph hyphenated to a Pegasus III time-of-flight mass spectrometer, LECO, St. Joseph, USA (Wagner et al. 2003). Chromatograms were acquired and processed by CHROMATOF software 1.00, Pegasus driver 1.61 (Leco; http://www.leco.de). Selective peak heights representing arbitrary mass spectral ion currents were normalized by the dry weight of the sample and to an internal standard, which was added upon extraction of the polar metabolite fraction. GC-TOF-MS chromatography data processing was performed using the TagFinder software (Luedemann et al. 2008). Metabolites were identified under manual supervision using the TagFinder, the NIST08 software (http://chemdata.nist.gov/) and the mass spectral and retention time index (RI) reference collection of the Golm Metabolome Database (Kopka et al. 2005; Hummel et al. 2010). Only metabolites identified in at least 4 of 7 replicates were considered. Wild-type (0 h) was used as reference condition for the calculation of $\log _{2}$ ratios of metabolites. PCA was performed using $R$ (www.r-project.org). Hierarchical clustering with Pearson correlation was performed using the software MultiExperiment Viewer (www.tm4.org/mev; version 27 4.8.1).

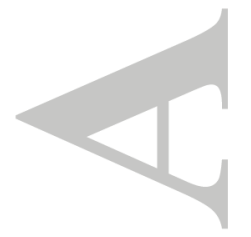




\section{RESULTS}

\section{Expression of AtPAO1-5 gene family members during salt stress}

To identify which AtPAO gene family members are transcriptionally responsive to salinity, we determined the expression of all five (AtPAO1-5) members by quantitative RT-PCR (qRT-PCR) in 4-week-old wild-type Arabidopsis plants treated with $50 \mathrm{mM} \mathrm{NaCl}$ between 0 $\mathrm{h}$ and $96 \mathrm{~h}$ (Fig. 1). The expression of most AtPAO genes (AtPAO1, AtPAO3, AtPAO4) remained unaltered by the saline treatment. In contrast, the expression of AtPAO5 progressively increased up to 4.5 -fold after $96 \mathrm{~h}$ of salt treatment. These analyses also revealed an early, but transient, peak of AtPAO2 expression after 3 h (Fig. 1). We concluded that AtPAO5 is the PAO family member that is most transcriptionally responsive to saline conditions in Arabidopsis, which might represent its potential involvement during salt stress.

\section{Salt-tolerance phenotype of atpao5-2 and atpao5-3 loss-of-function mutants}

To investigate the biological significance of the transcriptional activation of AtPAO5 by salt stress, two atpao5 T-DNA insertion mutants were isolated: atpao5-2 (Kim et al. 2014) and atpao5-3 (Fig. S1a). AtPAO5 expression was undetectable in both atpao5-2 and atpao5-3, indicating that they are loss-of-function mutants (Fig. S1b). Loss of AtPAO5 expression in atpao5-3 did not lead to consistent changes in the expression of AtPAO1-4 genes during salt stress (Fig. 1). These results point to the absence of transcriptional compensatory mechanisms between AtPAO5 and AtPAO1-4 gene family members during the saline treatment.

The salt-tolerant phenotype of atpao5-2, atpao5-3 and wild-type plants was investigated. Four-week-old plants grown in the absence of stress were exposed to gradual increases of $\mathrm{NaCl}$ (50 mM, $100 \mathrm{mM}, 150 \mathrm{mM}, 200 \mathrm{mM}$ and $250 \mathrm{mM} \mathrm{NaCl}$; see Materials and Methods). atpao5-2 and atpao5-3 mutants maintained higher \% of water content and chlorophyll levels than wild-type plants, consistent with a better performance under salt stress conditions (Fig. 2a - 2c). Salt stress tolerance was also phenotypically evident in atpao5-2 and atpao5-3 seedlings (Fig. 2d and 2e). We concluded that AtPAO5 loss-of-function leads to enhanced salt tolerance in Arabidopsis. 


\section{PA levels in atpao5 loss-of-function mutants during salt stress}

Free Put, Spd, Spm and tSpm levels were quantified in atpao5-2, atpao5-3 and wild-type plants exposed to $0,50,100$ and $150 \mathrm{mM} \mathrm{NaCl}$ for $48 \mathrm{~h}$ (Fig. 3). In absence of stress ( $0 \mathrm{mM}$ $\mathrm{NaCl}$ ), PA levels remained constant over the time of analysis (Fig. 3). Put levels significantly increased in the wild-type and both atpao5-2 and atpao5-3 mutant alleles exposed to $150 \mathrm{mM}$ $\mathrm{NaCl}$, while less or no Put increases were observed at lower $\mathrm{NaCl}$ concentrations $(50 \mathrm{mM}$ and $100 \mathrm{mM} \mathrm{NaCl}$, Fig. 3). Conversely, Spd levels declined in response to $100 \mathrm{mM}$ and $150 \mathrm{mM}$ $\mathrm{NaCl}$ in the wild-type and both atpao5 mutants (Fig. 3). No remarkable differences in the levels of Put, Spd or Spm were observed between atpao5 mutants and the wild-type (Fig. 3). However, the levels of tSpm were consistently higher in atpao5-2 and atpao5-3 than the wild-type, and remained unaltered during the saline treatment (Fig. 3). These results are in agreement with previous observations that $\mathrm{tSpm}$ is preferential substrate for AtPAO5 activity (Ahou et al. 2014; Kim et al. 2014). Since both atpao5-2 and atpao5-3 mutants exhibited identical salt tolerance phenotypes and PA profiles (Fig. 2 and 3), atpao5-3 was selected for further characterization.

\section{Sodium-ion levels in atpao5-3 during salt stress}

Quantification of $\mathrm{Na}^{+}$in leaves of atpao5-3 and wild-type during salt stress evidenced the occurrence of lower shoot sodium accumulation in atpao5-3 (Fig. S2). This observation suggested that $\mathrm{Na}^{+}$exclusion might contribute to the salt-tolerant phenotype of atpao5, which is in line with a potential role for PAs in regulating various ion channels (Pottosin \& Shabala 2014). However, since shoot $\mathrm{Na}^{+}$content in atpao5-3 almost reached wild-type levels after 96 h of $150 \mathrm{mM} \mathrm{NaCl}$ treatment, without evident symptoms of ion toxicity (Fig. 2 and S2), we reasoned that other mechanisms, in addition to transient $\mathrm{Na}^{+}$exclusion, might contribute to the salt tolerance phenotype induced by AtPAO5 loss-of-function.

\section{Quantification of ABA in atpao5-3 during salt stress}

ABA levels were determined during early (osmotic) and late (ionic, approximately $24 \mathrm{~h}$ to 48 $\mathrm{h}$ after $100 \mathrm{mM} \mathrm{NaCl}$ ) phases of salt treatment (Fig. 4a) (Munns \& Tester 2008). Under basal conditions, no differences in ABA contents were observed between atpao5-3 and wild-type plants. Treatment with $50 \mathrm{mM} \mathrm{NaCl}$ induced $\mathrm{ABA}$ accumulation in both genotypes after $3 \mathrm{~h}$ of treatment. However, ABA levels in atpao5-3 were moderately but significantly higher than the wild-type up to $48 \mathrm{~h}$ of salt treatment (Fig. 4a). Quantitative expression analyses of the ABA biosynthesis gene NCED3 and ABA-inducible gene RD29B (Uno et al., 2000) in 
atpao5-3 and wild-type correlated with ABA levels (Fig. 4b). Conversely, atpao5-3 accumulated less ABA than the wild-type at later stages of salt treatment (Fig. 4a). This observation is consistent with the salinity tolerance in atpao5-3 that is associated with delayed sodium accumulation in leaves (Fig. S2). We concluded that, although ABA biosynthesis is moderately promoted in atpao5-3 during the osmotic phase of salt stress, this is translated into higher ABA-dependent $R D 29 B$ gene expression compared with the wildtype.

\section{Hydrogen peroxide levels in atpao5-3 during salt stress}

To determine the ROS-detoxification capacity, hydrogen peroxide $\left(\mathrm{H}_{2} \mathrm{O}_{2}\right)$ levels in atpao5-3 and wild-type plants were quantified during salt stress (Fig. S3). Interestingly, $\mathrm{H}_{2} \mathrm{O}_{2}$ levels did not differ between both genotypes and accumulated to similar levels throughout the saline treatment (Fig. S3). Major increases in hydrogen peroxide were observed during the ionic phase of salt stress in both the wild-type and atpao5-3 (Fig. S3). These results suggest that the salt tolerance of the atpao5-3 mutant is not caused by enhanced ROS detoxification capacity. It also demonstrates that contribution of AtPAO5 to the overall generation of $\mathrm{H}_{2} \mathrm{O}_{2}$ during salt stress in Arabidopsis is rather limited.

\section{Transcriptome analyses of atpao5-3 during salt stress}

To get further insight into mechanisms underlying atpao5-3 salt tolerance, we determined the genome wide-expression profiles of atpao5-3 and wild-type plants before and after $3 \mathrm{~h}$ of 50 $\mathrm{mM} \mathrm{NaCl}$ treatment using Affymetrix microarrays. Genes that exhibited significant expression differences $(P<0.05$; fold change $\geq 2)$ were detected when comparing genotypes (atpao5-3 $0 \mathrm{~h}$ vs wild-type $0 \mathrm{~h}$, referred to as ' $p 0 w 0$ '; atpao5-3 $3 \mathrm{~h}$ vs wild-type $3 \mathrm{~h}$, ' $p 3 w 3$ ') and treatments (wild-type $3 \mathrm{~h} v s$ wild-type $0 \mathrm{~h}$, ' $w 3 w 0$ '; atpao5-3 $3 \mathrm{~h}$ vs atpao5-3 $0 \mathrm{~h}$, 'p3p0') (Fig. 5a; Tables S2-S15).

In the absence of stress, already 201 genes were differentially expressed between atpao5-3 and the wild-type ( $p 0 w 0$, Table S4). Gene-ontology analyses identified a significant number of genes related to signaling (Fig. 5b), which were mainly related to JA, protein kinases and transcription factors (Table S4). Interestingly, atpao5-3 exhibited higher basal expression of JA metabolism genes LOX4 (LIPOXYGENASE 4), AOC1 (ALLENE OXIDE CYCLASE 1), CYP94B3 (JASMONOYL-ISOLEUCINE-12-HYDROXYLASE), JAZ1 (JASMONATE ZIM- 
DOMAIN 1), JAZ5, JAZ6, JAZ7 transcriptional repressors, and the JAZ-target transcription factor MYC4 (Table S16). Overall, these results suggest that atpao5-3 has increased basal expression of several JA biosynthesis and signaling genes. Remarkably, most JA genes upregulated in atpao5-3 were also induced by salt stress in the wild-type ( $w 3 w 0$ in Table S16).

In the comparison between treatments ( $p 3 p 0$ and $w 3 w 0), 805$ differentially expressed genes in $p 3 p 0$ were detected and 622 in $w 3 w 0$, of which 356 were common between $p 3 p 0$ and w3w0 (Fig. 5a; Tables S2, S3 and S10). Common genes were similarly up- or downregulated and were mostly related to abiotic stimulus but also response to wounding, further reinforcing a potential role for JA on salt stress (Table S10; Fig. 5b). In the set of genes whose expression was exclusively changed in atpao5-3 by $50 \mathrm{mM} \mathrm{NaCl}$ treatment $(p 3 p 0$; Table S6), a significant enrichment of protein kinases was identified (Table S6; Fig. 5b). We also found that salt treatment led to increased expression of the $\mathrm{Na}^{+} /$pyruvate symporter BASS2 (At2g26900, BILE ACID:SODIUM SYMPORTER FAMILY PROTEIN 2) and $\mathrm{Na}^{+} / \mathrm{H}^{+}$ antiporter NHD1 (At3g19490, SODIUM:HYDROGEN ANTIPORTER 1) in atpao5-3, which are sodium transporters localized to the chloroplast envelope that mediate the plastidial influx or efflux of $\mathrm{Na}^{+}$, respectively (Furumoto et al. 2011; Müller et al. 2014) (Table S6). Enhanced expression of NHDI in atpao5-3 might contribute to its salt tolerance, since nhdl mutants exhibit reduced growth, less chlorophyll levels and lower photosynthetic performance due to decreased capacity of sodium export from chloroplasts (Müller et al. 2014). Interestingly, exogenous application of $100 \mu \mathrm{M}$ tSpm to 7-day-old wild-type seedlings quickly induced the expression of NHDl and this response was not triggered by Spm (Fig. S4). This early peak of NHD1 expression was followed by a 2.3-fold expression peak after 8 $\mathrm{h}$ of treatment with tSpm or Spm, which overlapped with BASS2 expression kinetics (Fig. S4). These data suggest that $\mathrm{tSpm}$ rapidly induces NHDl expression, thus potentially promoting $\mathrm{Na}^{+}$efflux from the chloroplast. Conversely, Spm and tSpm exhibit the same effect on BASS2 transcriptional upregulation after $8 \mathrm{~h}$ of exogenous treatment (Fig. S4).

Taken together, the data suggest that AtPAO5 loss-of-function impacts cell signaling through the transcriptional modulation of protein phosphorylation. This reinforces the hypothesis of a polyamine modulon in plants that involves transcriptional regulation of certain target genes by PAs (Tiburcio et al. 2014). 


\section{Transcriptional regulation of the JA pathway by tSpm. JA levels during salt stress.}

To investigate the effect of higher PAs on the transcriptional regulation of the JA pathway, we applied $100 \mu \mathrm{M}$ Spm or tSpm to 7-day-old wild-type seedlings and monitored the expression kinetics of JA biosynthesis genes ( $L O X 1, L O X 2, L O X 3, L O X 4, A O S, A O C 1$, AOC2, OPR3, OPCL1, CYP94B3; Fig. 6a), JA signaling genes (JAZ1, JAZ5, JAZ6, JAZ7, JAZ10, MYC2, MYC3 and MYC4; Fig. 6b) and JA response marker genes (VSP2 and PDF1.2; Fig. 6b). A significant upregulation of JA-marker gene JAZ10 was observed by exogenous application of tSpm, whereas Spm induced less, but still significant ( $\geq$ two-fold), upregulation. Conversely, PDF1.2 and VSP2 expression were only significantly induced by tSpm and not by Spm (Fig. 6b). Exogenously supplied tSpm also increased the expression of 13-lipooxygenases LOX1-4 (Fig. 6a). LOX encode enzymes oxidizing $\alpha$-linoleic acid to 13(S)-hydroperoxy-octadecatrienoic acid, which is precursor of different oxylipins. tSpm induced the transcriptional upregulation of LOX1 and LOX4 expression whereas Spm had little or no effect (Fig. 6a). The transcript levels of $A O S$, which is the first gene committed to JA biosynthesis, allene oxide cyclases AOC1 and AOC2, OPC-8:CoA ligase 1 (OPCL1) and CYP94B3 were also increased by tSpm treatment, and less by Spm. Conversely, OPR3 expression was more induced by Spm than tSpm (Fig. 6a).

Consistent with the transcriptional activation of the JA-pathway by tSpm, the expression of transcription factors $J A Z 1, J A Z 5, J A Z 6, J A Z 7$ and $J A Z 10$ was highly induced by exogenously supplied tSpm, and less by Spm (Fig. 6b). Among MYC2, MYC3 and MYC4 gene family members, tSpm significantly induced MYC4 expression after $24 \mathrm{~h}$ of treatment (Fig. 6b). Based on DNA binding specificity, MYC4 has been suggested to have different target genes than MYC2 (Fernández-Calvo et al. 2011). Remarkably, MYC4, ACL5 and AtPAO5 expression are found in the vasculature, which is the site of tSpm biosynthesis (FernándezCalvo et al. 2011; Kim et al. 2014).

Dose-response expression kinetics were determined for some $\mathrm{tSpm}$ and/or Spm induced genes after $15 \mathrm{~min}$ of treatment, using different concentrations of tSpm and Spm $(0,5,10,20$ and $100 \mu \mathrm{M}$; Fig. S5). Expression up-regulation was already observed using as low as $5 \mu \mathrm{M}$ tSpm or Spm, thus suggesting the specificity of this response (Fig. S5). Transcriptional responses increased linearly with the concentration of exogenously supplied PAs, with the highest differences observed at $100 \mu \mathrm{M}$ tSpm or Spm. 
Finally, and consistently with the transcriptional activation of the JA-pathway by tSpm, we observed higher levels of JA in atpao5-3 than in the wild-type during the saline treatment (Fig. 7). Overall, JA levels increased during saline stress, but accumulated to higher levels in atpao5-3 than the wild-type (Fig. 7).

Altogether, these results indicate that $\mathrm{tSpm}$, and to a lower extent Spm, promote the transcriptional activation of genes involved in JA biosynthesis and signaling. Furthermore, these data indicate that transcriptional upregulation of the JA pathway by PAs is isomerspecific with major contribution by $\mathrm{tSpm}$ and minor contribution by Spm.

\section{Global metabolite profiles of atpao5-3 during salt stress}

To get further insight into additional mechanisms underlying atpao5-3 salt stress tolerance from a metabolic view, we performed global metabolite profiling of atpao5-3 and wild-type plants during salt stress by gas chromatography-mass spectrometry (GC-MS). Our analyses identified 84 metabolites in 36 samples analyzed, which were annotated according to the MPIMP-Golm inventory list (Kopka et al. 2005) (Table S17). The chemical nature of 56 of such metabolites was known, and this set was used for Principal Component Analysis (PCA). For simplification, we used $0 \mathrm{~h}$ and $24 \mathrm{~h}$ time-points in which most metabolic changes were evident (Fig. 8a). PC1, which explains $72.8 \%$ of total variance, revealed the effect of salt stress on metabolic profiles. PC2 (13.3\% of total variance) reflected the differences between genotypes (atpao5-3 and wild-type), which increased after exposure to salt stress (Fig. 8a). atpao5-3 $0 \mathrm{~h}$ was shifted to the right in PC1, which suggests that atpao5-3 loss-of-function triggers a metabolic response similar to the salt treatment (Fig. 8a). Furthermore, metabolic responses to salt in atpao5-3 were earlier and more intense, consistent with a metabolic preacclimation hypothesis (PC1 in Fig. 8a). PC1 and PC2 loadings with highest values corresponded to galactinol and melibiose (Table S18). HCA Pearson's correlation and average linkage of metabolites and samples revealed the occurrence of four major metabolite clusters (Fig. 8b). Sample groupings evidenced contrasted differences between atpao5-3 and wild-type plants under salt stress. Heat map representation clearly indicated that some metabolic responses to salt were more intense in atpao5-3 than the wild-type, in line with PCA observations (Fig. 8b).

Based on metabolite profiles of the wild-type, we defined 'early' and 'late' salt-responsive metabolites. Late salt-responsive metabolites were those that exhibited significant $(P<0.05)$ 
differences in the wild-type after $24 \mathrm{~h}$ and $48 \mathrm{~h}$ of $150 \mathrm{mM} \mathrm{NaCl}$ treatment. Early saltresponsive metabolites corresponded to those showing significant $(P<0.05)$ differences in the wild-type after $8 \mathrm{~h}$ and $24 \mathrm{~h}$ of $50 \mathrm{mM} \mathrm{NaCl}$. The sum of Put and Agmatine levels was the only early, salt-responsive metabolite detected, but its levels were not consistently different between the wild-type and atpao5-3 during salt stress (Fig. S6). Conversely, 28 late saltresponsive metabolites were detected, of which 22 were increased and 6 reduced by salt treatment (Tables S19 and S17). Eighteen of the 28 late salt-responsive metabolites exhibited significant differences between atpao5-3 and wild-type (Table S19). Among these, we identified polyols (galactinol, myo-inositol), amino acids (proline, alanine, serine), TCA cycle intermediates (succinate, malate), sugars (lyxose), dehydroascorbic acid and shikimic acid (Fig. 9 and Table S17).

A number of additional metabolites were exclusively changed in atpao5-3 by salt stress and not in the wild-type. Such 'elicited' metabolites in atpao5-3 corresponded to amino acids (alanine), sugar alcohols (arabitol, erythritol), additional TCA cycle intermediates (fumarate, citrate), sugars (glucose, sucrose, rhamnose), and ethanolamine, which is precursor of choline and glycine-betaine (Chen \& Murata 2011) (Fig. 9 and Tables S20 and S17). Increased levels of glucose in atpao5-3 suggested activation of glycolysis that would feed the TCA cycle. Indeed, depletion of starch content and accumulation of soluble sugars seems to be a general response to salt stress (Kempa et al. 2008).

Altogether, metabolic profile analyses indicate that atpao5-3 mutant, which exhibits constitutively higher tSpm levels, responds earlier and more intense to salt stress, and accumulates higher levels of important compatible solutes. Overall, we conclude that tSpm triggers metabolic reprogramming in Arabidopsis that allows a faster and more intense response to salt stress. 


\section{DISCUSSION}

In this work, we have studied the potential involvement of the PA back-conversion pathway in Arabidopsis salt stress tolerance. Arabidopsis carries five PAO genes (AtPAO1-5) that encode proteins with distinct subcellular localizations and different substrate preferences. AtPAO1 and AtPAO5 are cytosolic enzymes, whereas AtPAO2-4 are localized in peroxisomes (Møller \& McPherson 1998; Cona et al. 2006; Reumann et al. 2009; PlanasPortell et al. 2013). Arabidopsis PAOs mediate PA back-conversion, which is coupled to $\mathrm{H}_{2} \mathrm{O}_{2}$ production. High levels of $\mathrm{H}_{2} \mathrm{O}_{2}$ generated by apoplastic PAO activity can trigger programmed cell death (PCD) under salt stress in tobacco (Moschou et al. 2008a; Moschou et al. 2008b). However, in Arabidopsis most PAO are peroxisomal and apoplastic oxidation seems to rely on $\mathrm{CuAO}$ activity (Planas et al. 2014). Among AtPAO gene family members, AtPAO5 is the most transcriptionally responsive to salt stress (Fig. 1). Consistent with a potential role for AtPAO5 on salt stress, two independent atpao5 loss-of-function mutants (atpao5-2 and atpao5-3) exhibit better performance under salinity (Fig. 2). Our results indicate that AtPAO5 activity is detrimental for salinity tolerance. Tolerance to abiotic stress has also been generated by antisense down-regulation of $P A O$ expression in tobacco (Moschou et al. 2008b). However, atpao5 tolerance does not correlate with diminished ROS production because the levels of $\mathrm{H}_{2} \mathrm{O}_{2}$ during salt stress are barely affected by atpao5 mutation (Fig. S3). Our analyses suggest that tSpm, which levels are constitutively higher in atpao5 mutants under salt stress (Fig. 3), triggers transcriptional and metabolic reprogramming that is isomer-specific. These results support an intrinsic role for PAs providing salt stress tolerance (Moschou et al, 2008b).

Hyperosmolarity is the primary effect of salt stress, causing turgor loss and ABA induction (Hasegawa 2013). To achieve osmotic adjustment, different compounds such as sugars, sugar alcohols, proline and glycine betaine accumulate (Chen \& Murata 2002). We observed that ABA biosynthesis is moderately promoted in atpao5-3 compared to the wild-type early during the osmotic phase of salt stress (Munns \& Tester 2008), but this is translated into significant differences in the expression of the ABA-inducible RD29B gene (Fig. 4) (Uno et al., 2000). Metabolic profiling by GC-MS of polar metabolites evidenced contrasted differences between atpao5-3 and wild-type plants during salt stress (Fig. 8a). Most metabolic changes were observed after $24 \mathrm{~h}$ of $\mathrm{NaCl}$ treatments and involved higher levels of sugars, polyols, proline and TCA cycle intermediates in atpao5-3 compared with the wildtype (Fig. 8b and 9). Most of such metabolites were also found increased in Arabidopsis 
wild-type plants exposed to $150 \mathrm{mM} \mathrm{NaCl}$ between $6 \mathrm{~h}$ to $5 \mathrm{~d}$ (Kempa et al. 2008). The higher levels of some osmoprotective compounds in atpao5-3 are compatible with early boost of ABA-biosynthesis (Kempa et al. 2008). Compatible solutes maintain cell turgor by osmotic adjustment, and redox balance by removing excess of ROS, thus contributing to abiotic stress tolerance (Munns \& Tester 2008; Székely et al. 2008; Krasensky \& Jonak 2012). Interactions between PAs and ABA have been described during drought and cold stresses, and lower ABA levels have been shown to underlie the freezing sensitivity of adcl mutants (Alcázar et al. 2006a; Cuevas et al. 2008b; Toumi et al. 2010). Conversely, ABA levels in atpao5-3 are lower than the wild-type at later stages of the saline treatment, which is consistent with better performance of atpao5-3 under salt stress that leads to reduced stress perception. Reduction in the levels of organic acids and TCA-cycle intermediates are often observed in metabolite profiling of glycophyte species exposed to saline stress (Gong et al. 2005; Sanchez et al. 2008; Hill et al. 2013; Zhao et al. 2014a). These observations suggest that energy production and/or plant growth are repressed by salinity (Zhao et al. 2014a). In this work, we have observed that atpao5-3 accumulates higher levels of TCA cycle intermediates during salt stress (Fig. 9) and this associates with tolerance. Higher levels of soluble sugars suggest activation of glycolysis that would feed the TCA cycle. Taken together, atpao5-3 mutation impacts cell metabolism through promotion of the accumulation of stress protective compounds and TCA cycle intermediates.

Despite salt stress tolerance was observed at later stages of treatment (Fig. 2), we determined transcriptional changes early during salt stress ( $3 \mathrm{~h}$ after $50 \mathrm{mM} \mathrm{NaCl}$ treatment; Fig. 5) to distinguish between direct effects from atpao5 mutation from late, possibly indirect, transcriptional changes derived from long-term salt exposure. Microarray-expression analyses revealed an enhanced transcriptional steady-state of the JA pathway in atpao5-3 mutants (Table S16). Quantitative expression of several JA-biosynthesis, signaling and marker genes from wild-type seedlings treated with $100 \mu \mathrm{M}$ tSpm or Spm evidenced that this effect is isomer-specific (Fig. 6). The expression of most JA-related genes analyzed responded to exogenous tSpm within 15 min of treatment, which is consistent with a direct effect of tSpm on transcriptional regulation. In addition, atpao5-3 exhibited higher JA levels than the wild-type during salt stress (Fig. 7). JAs are known to accumulate under saline conditions (Fig. 7) (Pedranzani et al.; Moons et al. 1997) and correlations between high JA levels and salinity tolerance have been observed in different rice cultivars (Kang et al. 2005). Furthermore, most transcriptionally up-regulated JA genes detected in the microarray were 
responsive to salt treatment (Table S16). Additional recent evidence support that JA are positive regulators of salt tolerance (Kazan, 2015; Zhao et al. 2014; Dong et al. 2013). Based on these evidences, we suggest that tSpm-triggered transcriptional upregulation of JA biosynthesis and signaling genes, as well as higher JA levels, contribute to the metabolic reprogramming and salt tolerance phenotypes of atpao5 mutants. We suggest that both ABA and JA pathways are involved in tSpm-triggered salt tolerance in Arabidopsis.

Microarray analyses also identified that the expression of BASS2 and NHD1 genes were upregulated by salt stress in atpao5-3. BASS2 encodes a plastid localized $\mathrm{Na}^{+} /$pyruvate symporter the activity of which is coupled with that of the $\mathrm{Na}^{+} / \mathrm{H}^{+}$antiporter NHD1 (Furumoto et al. 2011). BASS2 functions in C4 photosynthesis and provides pyruvate for the plastidic methylerythritol phosphate (MEP) pathway in C3 plants (Furumoto et al. 2011). In Arabidopsis, NHDl $\left(\mathrm{Na}^{+} / \mathrm{H}^{+}\right.$ANTIPORTER 1) mutation leads to higher plastidic $\mathrm{Na}^{+}$, which is associated with reduced growth and lower chlorophyll levels (Müller et al. 2014). Interestingly, tSpm rapidly induces NHD1 expression (Fig. S4) which may allow higher efflux of chloroplastic $\mathrm{Na}^{+}$, thus contributing to enhanced tolerance against salt stress. In addition, PAs with higher net charge have stronger inhibitory effect on the vacuolar nonselective cation channels, hence, potentially assisting vacuolar $\mathrm{Na}^{+}$sequestration (Pottosin \& Shabala 2014).

Altogether, we propose that mechanisms underlying salt-stress tolerance by PAs are diverse and additive, and involve transcriptional and metabolic reprogramming by $\mathrm{tSpm}$. This view is consistent with co-evolution of PAs with stress pathways that has allowed efficient integration of environmental signals into transcriptional and metabolic responses. Furthermore, we conclude that tSpm is not only involved in plant development, but also plays an important role in abiotic-stress tolerance. 


\section{Acknowledgments}

We thank Prof. Tomonobu Kusano for providing advice in the determination of thermospermine levels in Arabidopsis thaliana. Research in this work has been supported by the Spanish Ministerio de Ciencia e Innovación (BIO2011-29683 and CSD2007-00036) and the Generalitat de Catalunya (SGR2009-1060) to A.F.T. R.A. acknowledges support from the

Ramón y Cajal Program (RYC-2011-07847) of the Ministerio de Ciencia e Innovación (Spain).
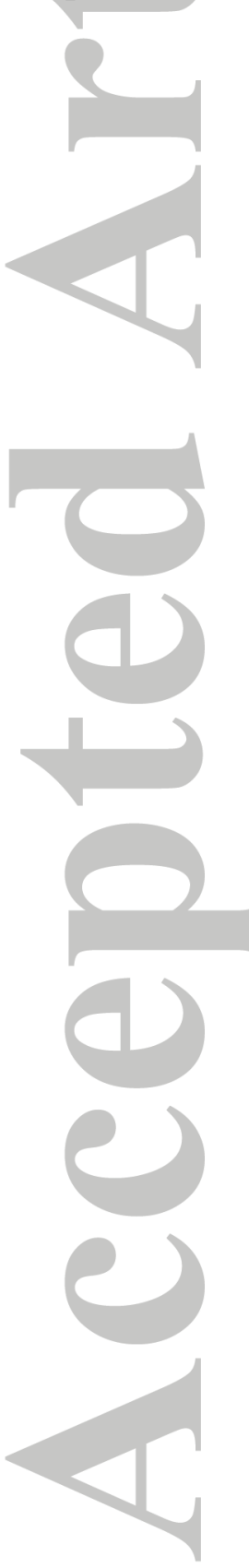


\section{REFERENCES}

Ahou A., Martignago D., Alabdallah O., Tavazza R., Stano P., Macone A., ..., Tavladoraki P. (2014) A plant spermine oxidase/dehydrogenase regulated by the proteasome and polyamines. Journal of Experimental Botany 65, 1585-1603.

Alcázar R., Altabella T., Marco F., Bortolotti C., Reymond M., Koncz C., .., Tiburcio A.F. (2010) Polyamines: molecules with regulatory functions in plant abiotic stress tolerance. Planta 231, $1237-1249$.

Alcázar R., Cuevas J.C., Patrón M., Altabella T. \& Tiburcio A.F. (2006a) Abscisic acid modulates polyamine metabolism under water stress in Arabidopsis thaliana. Physiologia Plantarum 128, $448-455$.

Alcázar R., García-Martínez J.L., Cuevas J.C., Tiburcio A.F. \& Altabella T. (2005) Overexpression of $A D C 2$ in Arabidopsis induces dwarfism and late-flowering through GA deficiency. The Plant Journal 43, 425-436.

Alcázar R., Marco F., Cuevas J.C., Patron M., Ferrando A., Carrasco P., ..., Altabella T. (2006b) Involvement of polyamines in plant response to abiotic stress. Biotechnology Letters 28, 18671876.

Alet A.I., Sánchez D.H., Cuevas J.C., Marina M., Carrasco P., Altabella T., ..., Ruiz O.A. (2012) New insights into the role of spermine in Arabidopsis thaliana under long-term salt stress. Plant Science 182, 94-100.

Bagni N., Ruiz-Carrasco K., Franceschetti M., Fornalè S., Fornasiero R.B. \& Tassoni A. (2006) Polyamine metabolism and biosynthetic gene expression in Arabidopsis thaliana under salt stress. Plant Physiology and Biochemistry 44, 776-786.

Bitrián M., Zarza X., Altabella T., Tiburcio A.F. \& Alcázar R. (2012) Polyamines under abiotic stress: metabolic crossroads and hormonal crosstalks in plants. Metabolites 2, 516-528.

Chen T.H. \& Murata N. (2002) Enhancement of tolerance of abiotic stress by metabolic engineering of betaines and other compatible solutes. Current Opinion in Plant Biology 5, 250-257.

Chen T.H.H. \& Murata N. (2011) Glycinebetaine protects plants against abiotic stress: mechanisms and biotechnological applications. Plant, Cell \& Environment 34, 1-20.

Cona A., Rea G., Angelini R., Federico R. \& Tavladoraki P. (2006) Functions of amine oxidases in plant development and defence. Trends in Plant Science 11, 80-88.

Cuevas J.C., López-Cobollo R., Alcázar R., Zarza X., Koncz C., Altabella T., ..,, Ferrando A. (2008b) Putrescine is involved in Arabidopsis freezing tolerance and cold acclimation by regulating abscisic acid levels in response to low temperature. Plant Physiology 148, 1094-1105.

Dong W., Wang M., Xu F., Quan T., Peng K., Xiao L. \& Xia G. (2013) Wheat oxophytodienoate reductase gene TaOPRI confers salinity tolerance via enhancement of abscisic acid signaling and reactive oxygen species scavenging. Plant Physiology 161, 1217-1228.

Durgbanshi A., Arbona V., Pozo O., Miersch O., Sancho J. V. \& Gómez-Cadenas A. (2005) Simultaneous determination of multiple phytohormones in plant extracts by liquid 
chromatography-electrospray tandem mass spectrometry. Journal of Agricultural and Food Chemistry 53, 8437-8442.

Erban A., Schauer N., Fernie A.R. \& Kopka J. (2007) Nonsupervised construction and application of mass spectral and retention time index libraries from time-of-flight gas chromatography-mass spectrometry metabolite profiles. Methods in Molecular Biology 358, 19-38.

Fernández-Calvo P., Chini A., Fernández-Barbero G., Chico J.-M., Gimenez-Ibanez S., Geerinck J., ..., Solano R. (2011) The Arabidopsis bHLH transcription factors MYC3 and MYC4 are targets of JAZ repressors and act additively with MYC2 in the activation of jasmonate responses. Plant Cell 23, $701-715$.

Fincato P., Moschou P.N., Spedaletti V., Tavazza R., Angelini R., Federico R., ..., Tavladoraki P. (2011) Functional diversity inside the Arabidopsis Polyamine Oxidase gene family. Journal of Experimental Botany 62, 1155-1168.

Furumoto T., Yamaguchi T., Ohshima-Ichie Y., Nakamura M., Tsuchida-Iwata Y., Shimamura M., ... Izui K. (2011) A plastidial sodium-dependent pyruvate transporter. Nature 476, 472-475.

Gaxiola R.A., Li J., Undurraga S., Dang L.M., Allen G.J., Alper S.L. \& Fink G.R. (2001) Droughtand salt-tolerant plants result from overexpression of the AVP1 $\mathrm{H}^{+}$-pump. Proceedings of the National Academy of Sciences of the United States of America 98, 11444-9.

Ghuge S.A., Carucci A., Rodrigues Pousada R.A., Tisi A., Franchi S., Tavladoraki P., ..., Cona A. (2015) The apoplastic copper amine oxidase AtAO1 mediates jasmonic acid-induced protoxylem differentiation in Arabidopsis roots. Plant Physiology 168, 690-707.

Gómez-Cadenas A., Pozo O.J., García-Augustín P. \& Sancho J. V. (2002) Direct analysis of abscisic acid in crude plant extracts by liquid chromatography--electrospray/tandem mass spectrometry. Phytochemical Analysis 13, 228-234.

Gong Q., Li P., Ma S., Indu Rupassara S. \& Bohnert H.J. (2005) Salinity stress adaptation competence in the extremophile Thellungiella halophila in comparison with its relative Arabidopsis thaliana. The Plant Journal 44, 826-839.

Hasegawa P.M. (2013) Sodium $\left(\mathrm{Na}^{+}\right)$homeostasis and salt tolerance of plants. Environmental and Experimental Botany 92, 19-31.

Hill C.B., Jha D., Bacic A., Tester M. \& Roessner U. (2013) Characterization of ion contents and metabolic responses to salt stress of different Arabidopsis AtHKT1;1 genotypes and their parental strains. Molecular Plant 6, 350-368.

Huang da W., Sherman B.T. \& Lempicki R.A. (2009) Systematic and integrative analysis of large gene lists using DAVID bioinformatics resources. Nature Protocols 4, 44-57.

Hummel J., Strehmel N., Selbig J., Walther D., \& Kopka J. (2010) Decision tree supported substructure prediction of metabolites from GC-MS profiles. Metabolomics 6, 322-333.

Kamada-Nobusada T., Hayashi M., Fukazawa M., Sakakibara H. \& Nishimura M. (2008) A putative peroxisomal polyamine oxidase, AtPAO4, is involved in polyamine catabolism in Arabidopsis thaliana. Plant and Cell Physiology 49, 1272-1282. 
Kang D.-J., Seo Y.-J., Lee J.-D., Ishii R., Kim K.U., Shin D.H., ..., Lee I.-J. (2005) Jasmonic acid differentially affects growth, ion uptake and abscisic acid concentration in salt-tolerant and saltsensitive rice cultivars. Journal of Agronomy and Crop Science 191, 273-282.

Kazan K. (2015) Diverse roles of jasmonates and ethylene in abiotic stress tolerance. Trends in Plant Science 20, 219-229.

Kempa S., Krasensky J., Dal Santo S., Kopka J. \& Jonak C. (2008) A central role of abscisic acid in stress-regulated carbohydrate metabolism. PloS One 3, e3935.

Kim D.W., Watanabe K., Murayama C., Izawa S., Niitsu M., Michael A.J., ..., Kusano T. (2014) Polyamine Oxidase5 regulates Arabidopsis growth through thermospermine oxidase activity. Plant Physiology 165, 1575-1590.

Kopka J., Schauer N., Krueger S., Birkemeyer C., Usadel B., Bergmüller E., ..., Steinhauser D. (2005) GMD@CSB.DB: the Golm Metabolome Database. Bioinformatics 21, 1635-1638.

Krasensky J. \& Jonak C. (2012) Drought, salt, and temperature stress-induced metabolic rearrangements and regulatory networks. Journal of Experimental Botany 63, 1593-1608.

Kusano T., Berberich T., Tateda C. \& Takahashi Y. (2008) Polyamines: essential factors for growth and survival. Planta 228, 367-381.

Loreto F. \& Velikova V. (2001) Isoprene produced by leaves protects the photosynthetic apparatus against ozone damage, quenches ozone products, and reduces lipid peroxidation of cellular membranes. Plant Physiology 127, 1781-1787.

Luedemann A., Strassburg K., Erban A. \& Kopka J. (2008) TagFinder for the quantitative analysis of gas chromatography--mass spectrometry (GC-MS)-based metabolite profiling experiments. Bioinformatics 24, 732-737.

Møller S.G. \& McPherson M.J. (1998) Developmental expression and biochemical analysis of the Arabidopsis ATAOl gene encoding an $\mathrm{H}_{2} \mathrm{O}_{2}$-generating diamine oxidase. The Plant Journal 13, 781-791.

Moons A., Prinsen E., Bauw G. \& Van Montagu M. (1997) Antagonistic effects of abscisic acid and jasmonates on salt stress-inducible transcripts in rice roots. Plant Cell 9, 2243-2259.

Moschou P.N., Delis I.D., Paschalidis K.A. \& Roubelakis-Angelakis K.A. (2008a) Transgenic tobacco plants overexpressing Polyamine Oxidase are not able to cope with oxidative burst generated by abiotic factors. Physiologia Plantarum 133, 140-156.

Moschou P.N., Paschalidis K. a, Delis I.D., Andriopoulou A.H., Lagiotis G.D., Yakoumakis D.I. \& Roubelakis-Angelakis K.A. (2008b) Spermidine exodus and oxidation in the apoplast induced by abiotic stress is responsible for $\mathrm{H}_{2} \mathrm{O}_{2}$ signatures that direct tolerance responses in tobacco. Plant Cell 20, 1708-1724.

Moschou P.N., Sanmartin M., Andriopoulou A.H., Rojo E., Sanchez-Serrano J.J. \& RoubelakisAngelakis K.A. (2008c) Bridging the gap between plant and mammalian polyamine catabolism: a novel peroxisomal polyamine oxidase responsible for a full back-conversion pathway in Arabidopsis. Plant Physiology 147, 1845-1857. 
Moschou P.N., Wu J., Cona A., Tavladoraki P., Angelini R. \& Roubelakis-Angelakis K.A. (2012) The polyamines and their catabolic products are significant players in the turnover of nitrogenous molecules in plants. Journal of Experimental Botany 63, 5003-5015.

Müller M., Kunz H.-H., Schroeder J.I., Kemp G., Young H.S. \& Neuhaus H.E. (2014) Decreased capacity for sodium export out of Arabidopsis chloroplasts impairs salt tolerance, photosynthesis and plant performance. The Plant Journal 78, 646-658.

Munns R. \& Tester M. (2008) Mechanisms of salinity tolerance. Annual Review of Plant Biology 59, 651-681.

Naka Y., Watanabe K., Sagor G.H.M., Niitsu M., Pillai M.A., Kusano T. \& Takahashi Y. (2010) Quantitative analysis of plant polyamines including thermospermine during growth and salinity stress. Plant Physiology and Biochemistry 48, 527-533.

Pedranzani H., Racagni G., Alemano S., Miersch O., Ramírez I., Peña-Cortés H., ..., Abdala G. Salt tolerant tomato plants show increased levels of jasmonic acid. Plant Growth Regulation 41, 149158.

Porra R.J. (2002) The chequered history of the development and use of simultaneous equations for the accurate determination of chlorophylls $a$ and $b$. Photosynthesis Research 73, 149-156.

Pottosin I. \& Shabala S. (2014) Polyamines control of cation transport across plant membranes: implications for ion homeostasis and abiotic stress signaling. Frontiers in Plant Science 5, 154.

Pottosin I., Velarde-Buendía A.M., Bose J., Fuglsang A.T. \& Shabala S. (2014) Polyamines cause plasma membrane depolarization, activate $\mathrm{Ca}$-, and modulate H-ATPase pump activity in pea roots. Journal of Experimental Botany 65, 2463-2472.

Reumann S., Quan S., Aung K., Yang P., Manandhar-Shrestha K., Holbrook D., ..., Hu J. (2009) Indepth proteome analysis of Arabidopsis leaf peroxisomes combined with in vivo subcellular targeting verification indicates novel metabolic and regulatory functions of peroxisomes. Plant Physiology 150, 125-143.

Saha J., Brauer E.K., Sengupta A., Popescu S.C., Gupta K. \& Gupta B. (2015) Polyamines as redox homeostasis regulators during salt stress in plants. Frontiers in Environmental Science 3, 1-13.

Sánchez D.H., Siahpoosh M.R., Roessner U., Udvardi M. \& Kopka J. (2008) Plant metabolomics reveals conserved and divergent metabolic responses to salinity. Physiologia Plantarum 132, 209 219.

Székely G., Abrahám E., Cséplo A., Rigó G., Zsigmond L., Csiszár J., ..., Szabados L. (2008) Duplicated P5CS genes of Arabidopsis play distinct roles in stress regulation and developmental control of proline biosynthesis. The Plant Journal 53, 11-28.

Takahashi T. \& Kakehi J.-I. (2010) Polyamines: ubiquitous polycations with unique roles in growth and stress responses. Annals of Botany 105, 1-6.

Takahashi Y., Cong R., Sagor G.H.M., Niitsu M., Berberich T. \& Kusano T. (2010) Characterization of five polyamine oxidase isoforms in Arabidopsis thaliana. Plant Cell Reports 29, 955-965.

Takano A., Kakehi J.-I. \& Takahashi T. (2012) Thermospermine is not a minor polyamine in the plant kingdom. Plant and Cell Physiology 53, 606-616. 
Tanou G., Ziogas V., Belghazi M., Christou A., Filippou P., Job D., Fotopoulus V. \& Molassiotis A. (2014). Polyamines reprogram oxidative and nitrosative status and the proteome of citrus plants exposed to salinity stress. Plant Cell \& Environment 37, 864-885.

Tassoni A., Franceschetti M. \& Bagni N. (2008) Polyamines and salt stress response and tolerance in Arabidopsis thaliana flowers. Plant Physiology and Biochemistry 46, 607-613.

Tavladoraki P., Cona A., Federico R., Tempera G., Viceconte N., Saccoccio S., ..., Agostinelli E. (2012) Polyamine catabolism: target for antiproliferative therapies in animals and stress tolerance strategies in plants. Amino acids 42, 411-426.

Tavladoraki P., Rossi M.N., Saccuti G., Perez-Amador M.A., Polticelli F., Angelini R. \& Federico R. (2006) Heterologous expression and biochemical characterization of a polyamine oxidase from Arabidopsis involved in polyamine back conversion. Plant Physiology 141, 1519-1532.

Tiburcio A.F., Altabella T., Bitrián M. \& Alcázar R. (2014) The roles of polyamines during the lifespan of plants: from development to stress. Planta 240, 1-18.

Tong W., Yoshimoto K., Kakehi J.-I., Motose H., Niitsu M. \& Takahashi T. (2014) Thermospermine modulates expression of auxin-related genes in Arabidopsis. Frontiers in Plant Science 5, 94.

Toumi I., Moschou P.N., Paschalidis K.A., Bouamama B., Ben Salem-Fnayou A., Ghorbel A.W., ..., Roubelakis-Angelakis K.A. (2010) Abscisic acid signals reorientation of polyamine metabolism to orchestrate stress responses via the polyamine exodus pathway in grapevine. Journal of Plant Physiology 167, 519-525.

Uno Y., Furihata T., Abe H., Yoshida R., Shinozaki K. \& Yamaguchi-Shinozaki K. (2000) Arabidopsis basic leucine zipper transcription factors involved in an abscisic acid-dependent signal transduction pathway under drought and high-salinity conditions. Proceedings of the National Academy of Sciences of the United States of America 97, 11632-11637.

Urano K., Yoshiba Y., Nanjo T., Igarashi Y., Seki M., Sekiguchi F., .., Shinozaki K. (2003) Characterization of Arabidopsis genes involved in biosynthesis of polyamines in abiotic stress responses and developmental stages. Plant, Cell \& Environment 26, 1917-1926.

Vera-Sirera F., Minguet E.G., Singh S.K., Ljung K., Tuominen H., Blázquez M.A. \& Carbonell J. (2010) Role of polyamines in plant vascular development. Plant Physiology and Biochemistry 48, 534-539.

Wagner C., Sefkow M. \& Kopka J. (2003) Construction and application of a mass spectral and retention time index database generated from plant GC/EI-TOF-MS metabolite profiles. Phytochemistry 62, 887-900.

Yamaguchi K., Takahashi Y., Berberich T., Imai A., Miyazaki A., Takahashi T., .., Kusano T. (2006) The polyamine spermine protects against high salt stress in Arabidopsis thaliana. FEBS Letters 580, 6783-6788.

Zhao X., Wang W., Zhang F., Deng J., Li Z. \& Fu B. (2014a) Comparative metabolite profiling of two rice genotypes with contrasting salt stress tolerance at the seedling stage. PLoS One 9, e108020. 
Zhao Y., Dong W., Zhang N., Ai X., Wang M., Huang Z., ..., Xia G. (2014b) A wheat allene oxide cyclase gene enhances salinity tolerance via jasmonate signaling. Plant Physiology 164, 10681076.
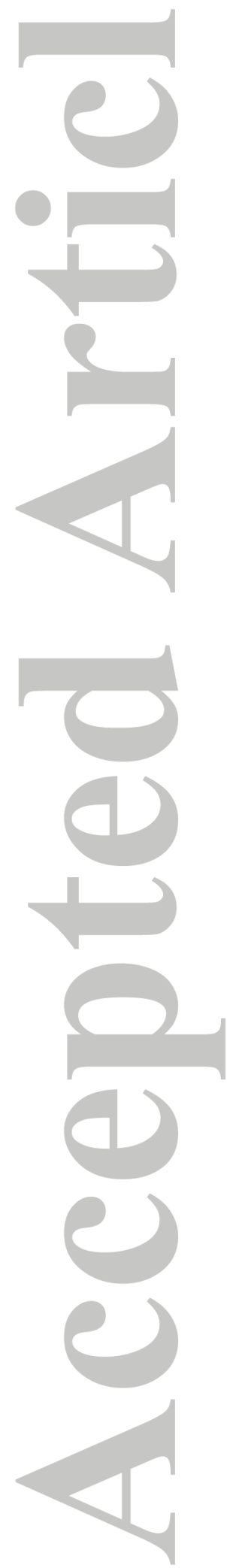

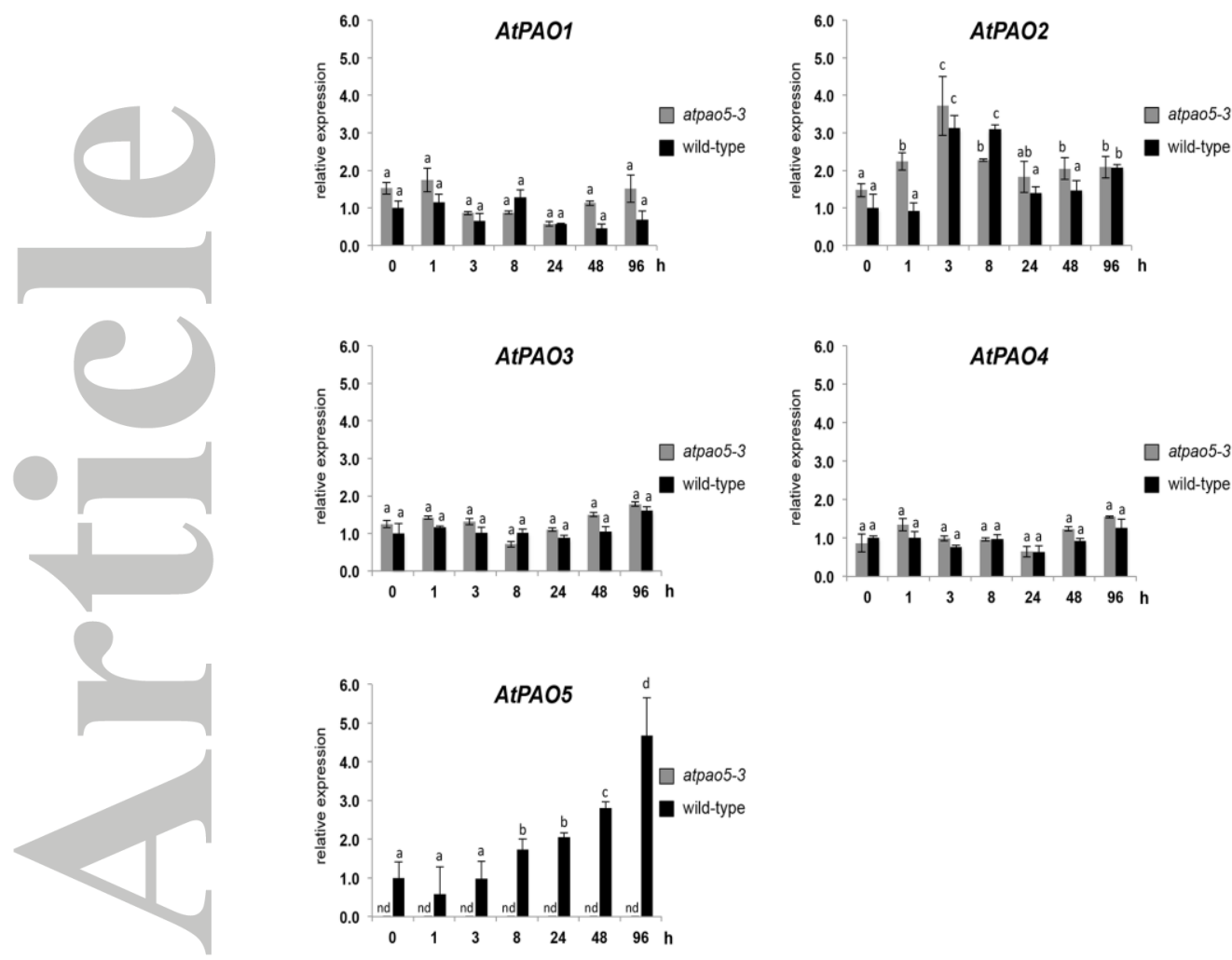

Figure 1. Transcript profiles of AtPAO gene family members during salt stress. Quantitative real-time PCR (qRT-PCR) expression analyses of AtPAO1-5 genes in 4-week-old Arabidopsis wild-type and atpao5-3 loss-of-function mutants exposed to $50 \mathrm{mM} \mathrm{NaCl}$ for 0 $96 \mathrm{~h}$. Values are relative to wild-type $(0 \mathrm{~h})$ and are the mean \pm SD of three biological replicates each with three technical replicates. nd, not detectable. Letters indicate values that are significantly different according to Student-Newman-Keuls test at $P$-value $<0.05$. 


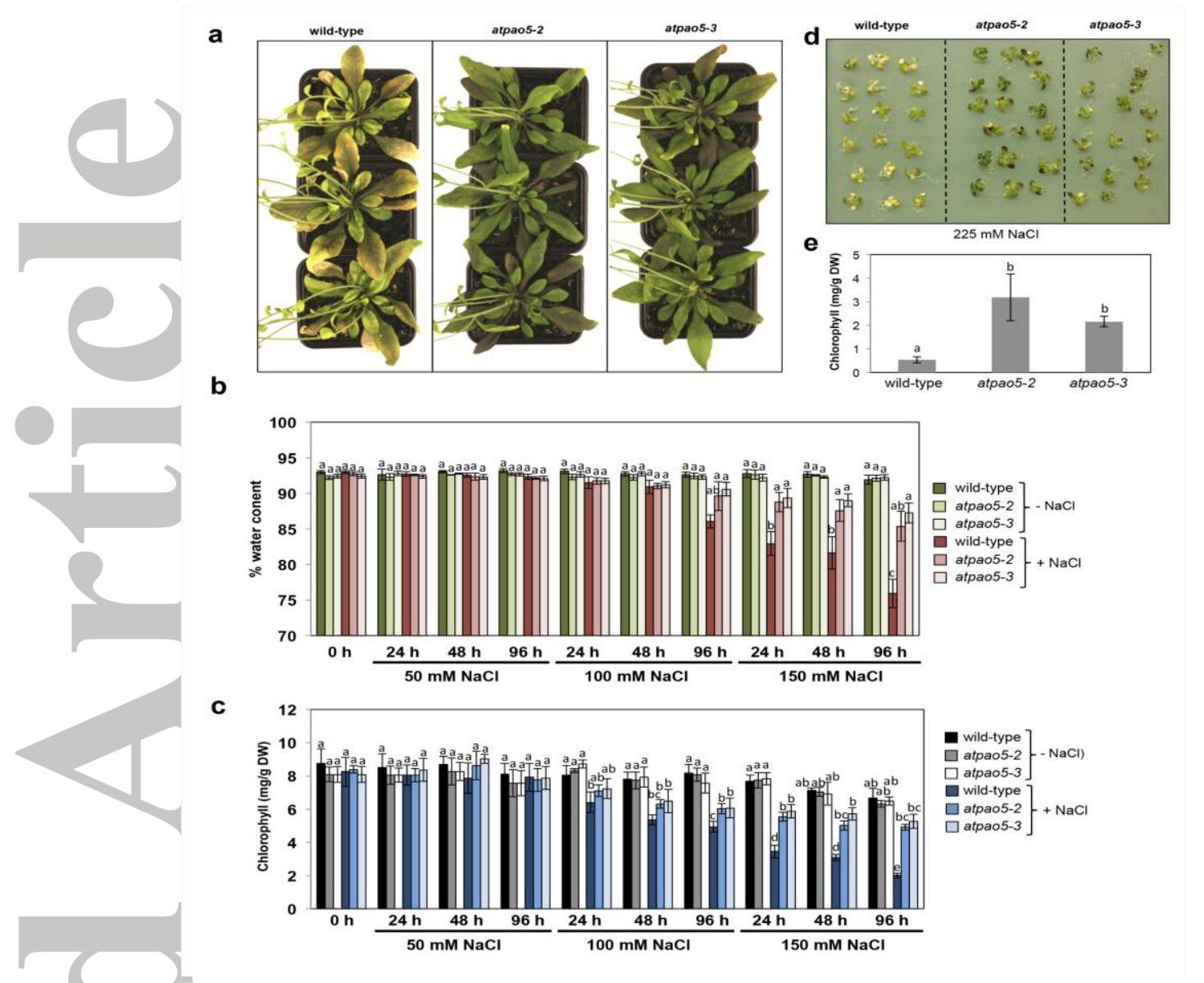

Figure 2. Salt stress tolerance of atpao5 compared to wild-type plants. (a) Phenotype of atpao5-2, atpao5-3 and wild-type plants after $96 \mathrm{~h}$ of $200 \mathrm{mM} \mathrm{NaCl}$ treatment with gradual increases of salt (see Materials and Methods). (b) \% of water content and (c) chlorophyll levels in atpao5-2, atpao5-3 and wild-type plants exposed to gradual increases of salt (+ $\mathrm{NaCl})$ and untreated controls (- $\mathrm{NaCl})$. (d) Phenotype and (e) chlorophyll levels of atpao5-2, atpao5-3 and wild-type seedlings exposed to gradual increases of salt (see Materials and Methods). Letters indicate values that are significantly different according to StudentNewman-Keuls test at $P$-value $<0.05$. 


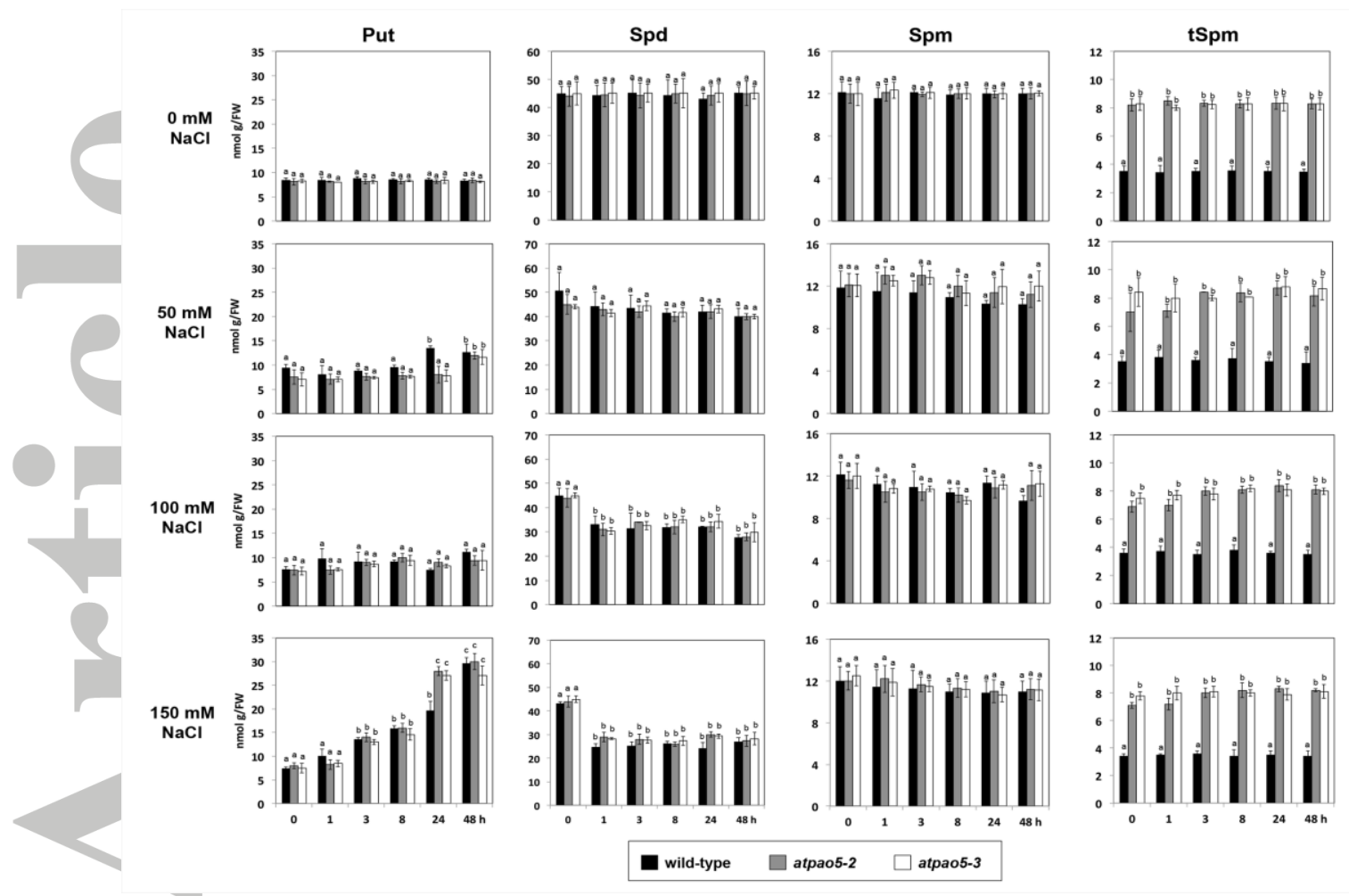

Figure 3. Free Put, Spd, Spm and tSpm levels in 4-week-old wild-type, atpao5-2 and atpao53 mutants treated with $0,50,100$ and $150 \mathrm{mM} \mathrm{NaCl}$ between 0 and $48 \mathrm{~h}$. Values are the mean \pm SD of three biological replicates each with three technical replicates. FW, fresh weight. Letters indicate values that are significantly different according to Student-Newman-Keuls test at $P$-value $<0.05$. 
a

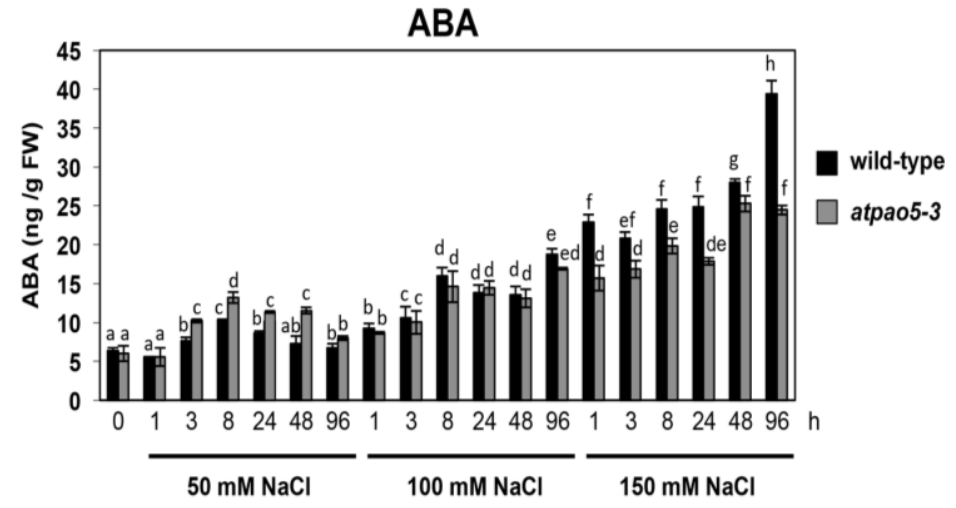

b
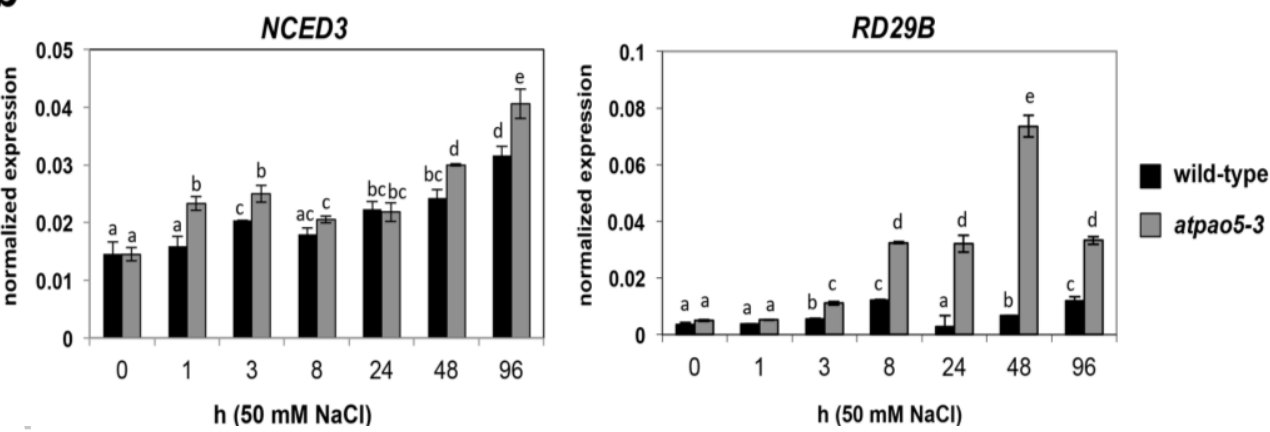

Figure 4. (a) Determination of ABA levels in atpao5-3 and wild-type plants exposed to gradual increases of $\mathrm{NaCl}$ between $50 \mathrm{mM}$ to $150 \mathrm{mM}$. Values are the mean $\pm \mathrm{SD}$ of five technical replicates. The analysis was repeated in two additional independent experiments showing similar results. (b) Quantitation of NCED3 and RD29B expression in atpao5-3 and wild-type plants exposed to $50 \mathrm{mM} \mathrm{NaCl}$ between $0-96 \mathrm{~h}$. Values are the mean $\pm \mathrm{SD}$ from three biological replicates each with three technical replicates. Letters indicate values that are significantly different according to Student-Newman-Keuls test at $P$-value $<0.05$. 
a

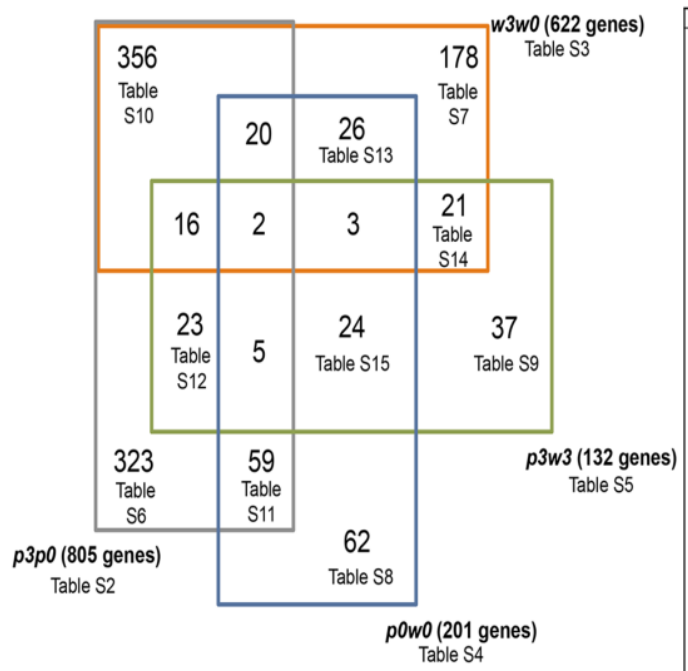

b

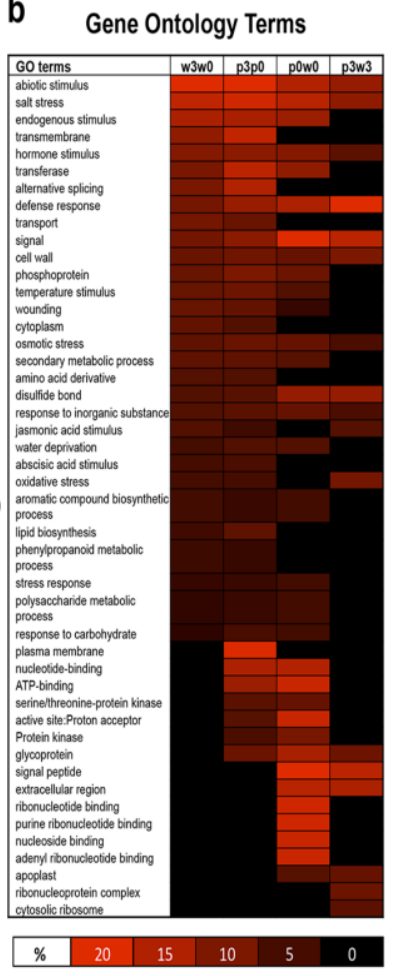

Figure 5. (a) Venn diagrams and (b) heat map of gene ontology terms from differentially expressed genes in atpao5-3 and wild-type plants treated with $50 \mathrm{mM} \mathrm{NaCl}$ between $0 \mathrm{~h}$ and $3 \mathrm{~h}$. Genes in the different comparisons ( $w 3 w 0$, wild-type $3 \mathrm{~h} v s$ wild-type $0 \mathrm{~h}$ of $50 \mathrm{mM} \mathrm{NaCl}$ treatment; $p 3 p 0$, atpao5-3 $3 \mathrm{~h}$ vs atpao5-3 0h of $50 \mathrm{mM} \mathrm{NaCl}$ treatment; p0w0, atpao5-3 $0 \mathrm{~h}$ vs wild-type $0 \mathrm{~h}$; and p3w3, atpao5-3 3h vs wild-type $3 \mathrm{~h}$ ) are listed in Tables S2-S15. 

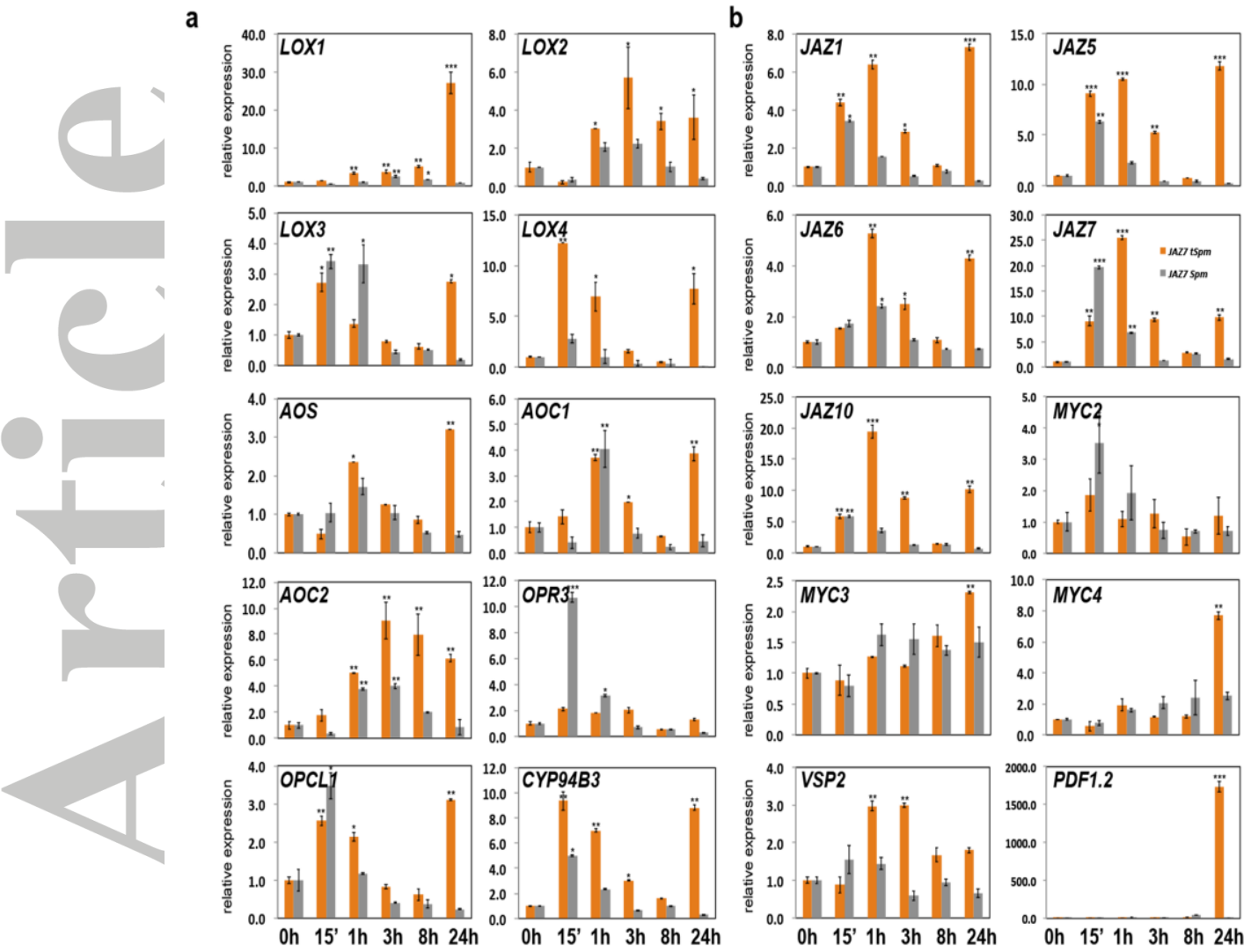

$\square \mathrm{tSpm} 100 \mu \mathrm{M}$

$\square \mathrm{Spm} 100 \mu \mathrm{M}$

Figure 6. Quantitative real-time gene expression analyses of JA-biosynthesis (a) and signaling (b) genes from 7-days-old wild-type seedlings treated with $100 \mu \mathrm{M}$ tSpm or 100 $\mu \mathrm{M}$ Spm during 0-24 h. Values are the mean from three biological replicates \pm SD each with three technical replicates. Significant differences in gene expression relative to time $0 \mathrm{~h}$ were determined using Student's t-test and indicated by asterisks: $* \mathrm{P}<0.05, * * \mathrm{P}<0.01, * * * \mathrm{P}<0.005$. 


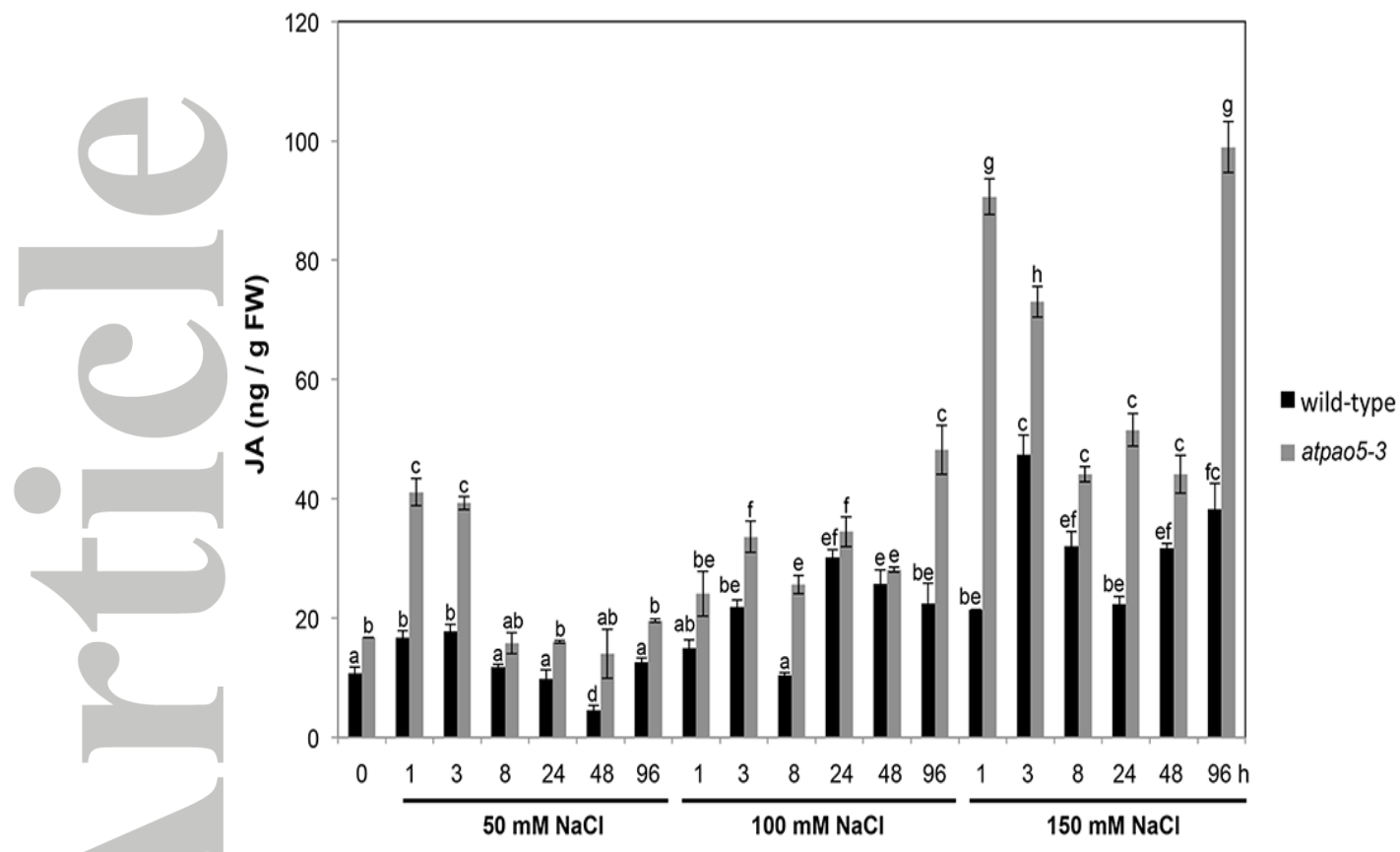

Figure 7. Quantification of JA levels in atpao5-3 and wild-type plants exposed to gradual increases of $\mathrm{NaCl}$ between $50 \mathrm{mM}$ to $150 \mathrm{mM}$. Values are the mean $\pm \mathrm{SD}$ of five technical replicates. The analysis was repeated in two additional independent experiments showing similar results. Letters indicate values that are significantly different according to StudentNewman-Keuls test at $P$-value $<0.05$. 


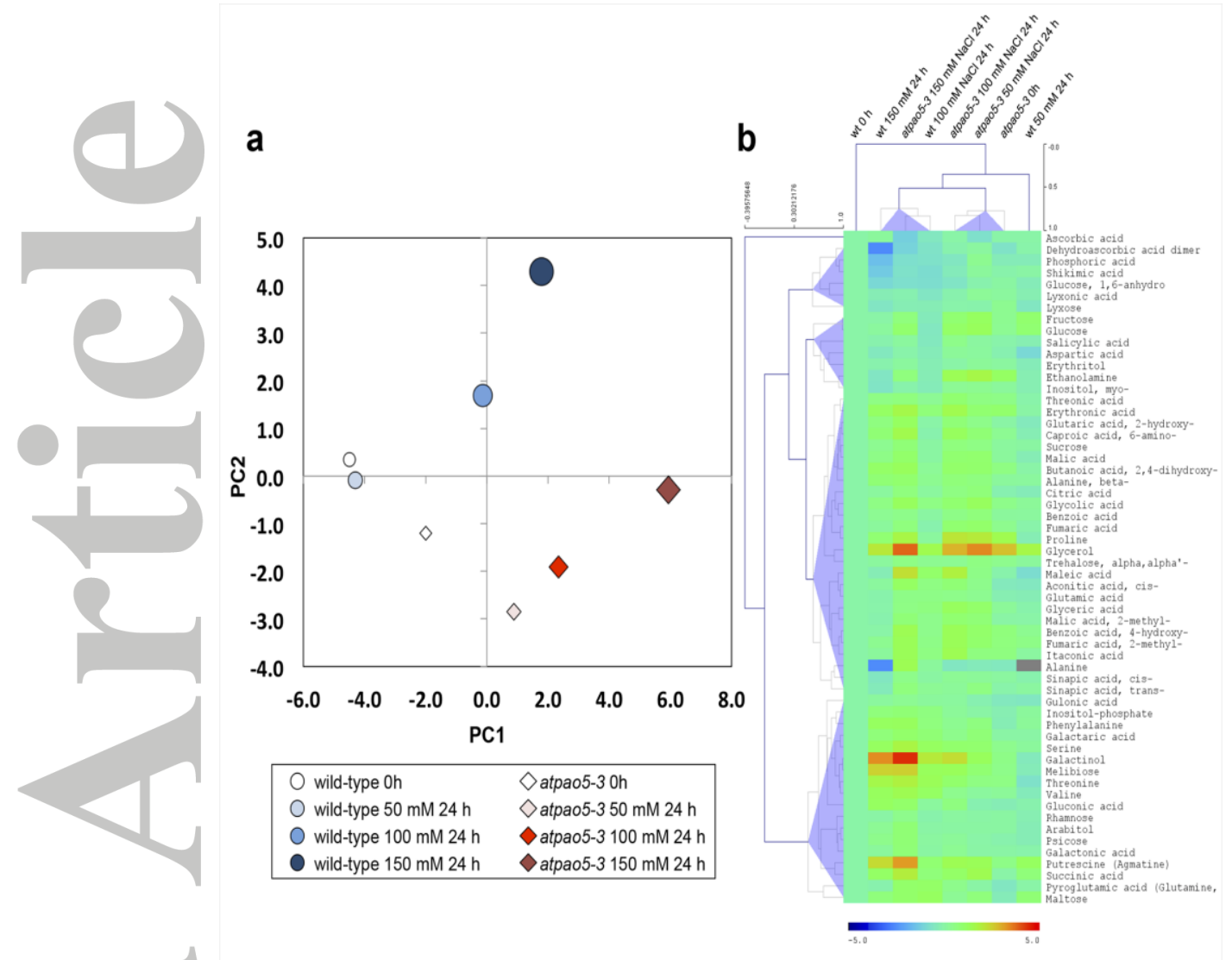

Figure 8. (a) Principal component analysis (PCA) and (b) HCA Pearson's correlation and average linkage of metabolites and samples from atpao5-3 and wild-type plants exposed to salt stress. 


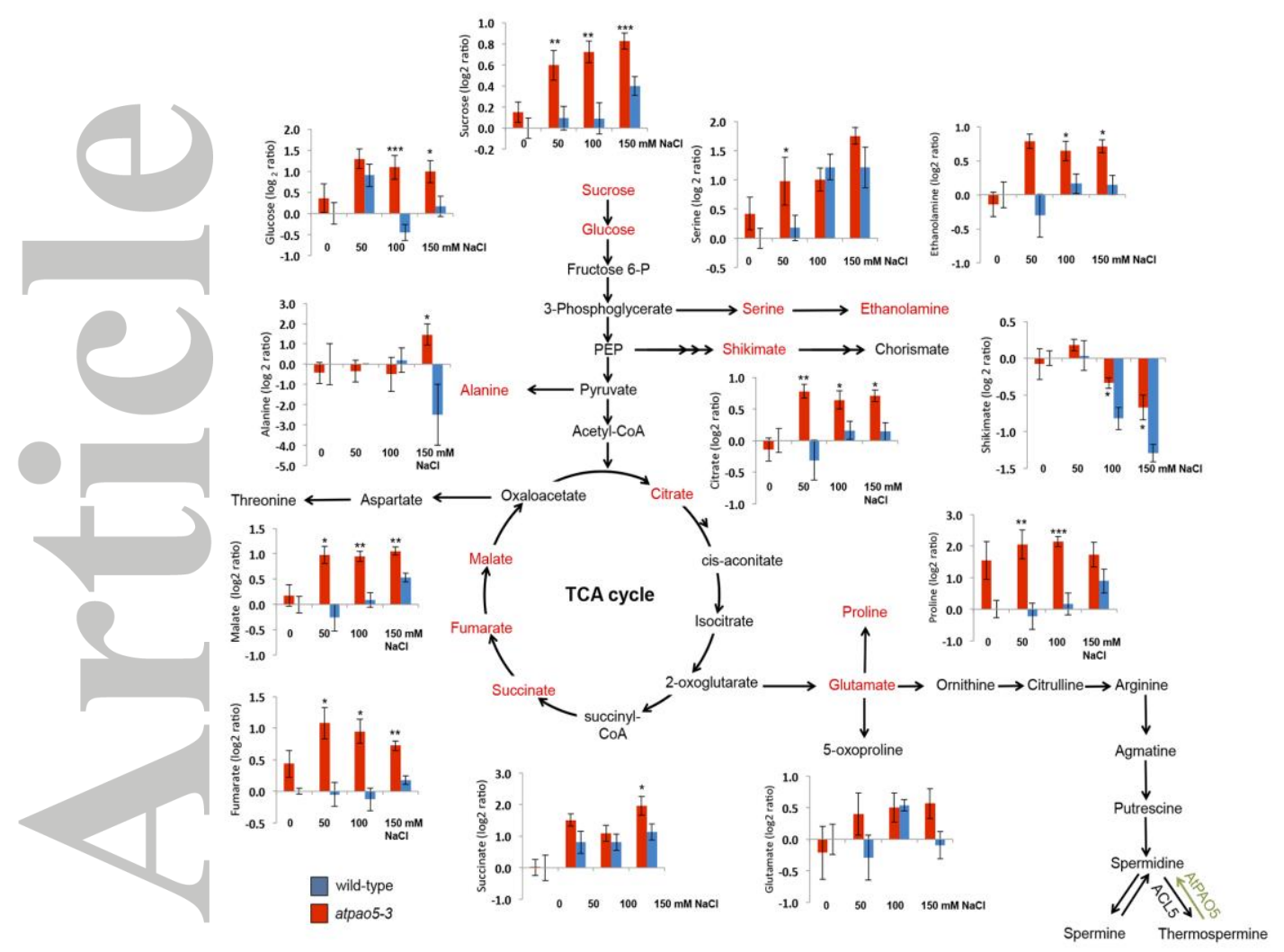

Figure 9. Schematic representation of TCA cycle intermediates and primary metabolites in associated pathways. $\log _{2}$ ratios $\pm \mathrm{SE}$ values are relative to wild-type $0 \mathrm{~h}$. Values are shown for atpao5-3 (red) and wild-type (blue) $24 \mathrm{~h}$ after 50, 100 and $150 \mathrm{mM} \mathrm{NaCl}$ treatment. Significant differences between genotypes at the same time point and salt concentration were determined using Student's t-test and indicated by asterisks: $* \mathrm{P}<0.05, * * \mathrm{P}<0.01, * * * \mathrm{P}<0.005$. 


\section{Figure legends Supplementary Figures S1- S6}

Figure S1. (a) Schematic representation of T-DNA insertion positions in atpao5-2 and atpao5-3 mutants. The AtPAO5 gene carries a single exon (highlighted in black). 5' and 3' uORFs are shown in gray. (b) RT-PCR expression analysis of AtPAO5 transcript levels in wild-type, atpao5-2 and atpao5-3 homozygous mutants. ACTIN2 was used as housekeeping gene.

Figure S2. Quantitation of sodium levels in leaves of atpao5-3 and wild-type plants exposed to gradual increases of $\mathrm{NaCl}$ from $50 \mathrm{mM}$ to $150 \mathrm{mM}$. Values are the mean $\pm \mathrm{SD}$ of five technical replicates. The experiment was repeated three times with similar results.

Figure S3. Hydrogen peroxide levels in atpao5-3 and wild-type plants exposed to gradual increases of $\mathrm{NaCl}$ between $50 \mathrm{mM}$ to $150 \mathrm{mM}$. Values are the mean $\pm \mathrm{SD}$ of five technical replicates. The analysis was repeated in two additional independent experiments showing similar results.

Figure S4. Quantitative real-time gene expression analyses of BASS2 and NHD1 genes from 7-days-old wild-type seedlings treated with $100 \mu \mathrm{M}$ tSpm or $100 \mu \mathrm{M}$ Spm during 0-24 h. Values are the mean from three biological replicates \pm SD each with three technical replicates. Letters indicate values that are significantly different according to StudentNewman-Keuls test at $P$-value $<0.05$.

Figure S5. Quantitative real-time gene expression analyses of JA-biosynthesis (LOX4 and $O P C L 1$ ) and signaling (JAZ5) genes from 12-days-old wild-type seedlings treated for 15 min with different concentrations $(0,5,10,20$ and $100 \mu \mathrm{M})$ of tSpm or Spm. Values are the mean from three biological replicates \pm SD each with three technical replicates. Letters indicate values that are significantly different according to Student-Newman-Keuls test at $P$-value $<0.05$.

Figure S6. Sum of Putrescine and Agmatine levels in atpao5-3 and wild-type plants exposed to salt stress. $\log _{2}$ ratios $\pm \mathrm{SE}$ are relative to wild-type $0 \mathrm{~h}$. 


\section{Supplementary Tables S1-S20}

Table S1. List of oligonucleotides used in this work.

Table S2. Genes differentially expressed in the comparison between atpao5-3 $3 \mathrm{~h}$ and atpao5-3 $0 \mathrm{~h}$ of $50 \mathrm{mM} \mathrm{NaCl}$ treatment (p3p0)

Table S3. Genes differentially expressed in the comparison between wild-type $3 \mathrm{~h}$ and wild-type $0 \mathrm{~h}$ of $50 \mathrm{mM} \mathrm{NaCl}$ treatment (w3w0)

Table S4. Genes differentially expressed in the comparison between atpao5-3 0h and wildtype $0 \mathrm{~h}(\mathrm{p} 0 \mathrm{w} 0)$

Table S5. Genes differentially expressed in the comparison between atpao5-3 $3 \mathrm{~h}$ and wildtype $3 \mathrm{~h}$ of $50 \mathrm{mM} \mathrm{NaCl}$ treatment (p3w3)

Table S6. Genes differentially expressed only in the comparison between atpao5-3 $3 \mathrm{~h}$ and atpao5-3 $0 \mathrm{~h}$ of $50 \mathrm{mM} \mathrm{NaCl}$ treatment (p3p0 only)

Table S7. Genes differentially expressed only in the comparison between wild-type $3 \mathrm{~h}$ and wild-type $0 \mathrm{~h}$ of $50 \mathrm{mM} \mathrm{NaCl}$ treatment (w3w0 only)

Table S8. Genes differentially expressed only in the comparison between atpao5-3 $0 \mathrm{~h}$ and wild-type $0 \mathrm{~h}$ of $50 \mathrm{mM} \mathrm{NaCl}$ treatment (p0w0 only)

Table S9. Genes differentially expressed only in the comparison between atpao5-3 $3 \mathrm{~h}$ vs wild-type $3 \mathrm{~h}$ of $50 \mathrm{mM} \mathrm{NaCl}$ treatment (p3w3 only)

Table S10. Genes differentially expressed and common between: p3p0 \& w3w0

Table S11. Genes differentially expressed and common between: $\mathrm{p} 3 \mathrm{p} 0$ \& p0w0

Table S12. Genes differentially expressed and common between: p3p0 \& p3w3

Table S13. Genes differentially expressed and common between: w3w0 \& p0w0

Table S14. Genes differentially expressed and common between: w3w0 \& p3w3

Table S15. Genes differentially expressed and common between: p0w0 \& p3w3

Table S16. Microarray analysis of JA biosynthesis and signaling genes in the comparisons p0w0, p3w3, w3w0 and p3p0.

Table S17. Metabolite analysis of atpao5-3 and wild-type plants exposed to $\mathrm{NaCl}$ stress.

Table S18. PC1 and PC2 loadings of wild-type and atpao5-3 metabolite profiles $24 \mathrm{~h}$ after treatment with $0,50,100$ and $150 \mathrm{mM} \mathrm{NaCl}$.

Table S19. Late salt responsive metabolites in wild-type. Metabolites exhibiting significant differences between wild-type and atpao5-3 are highlighted.

Table S20. List of metabolites differentially accumulated in atpao5-3 in response to salt 
stress.

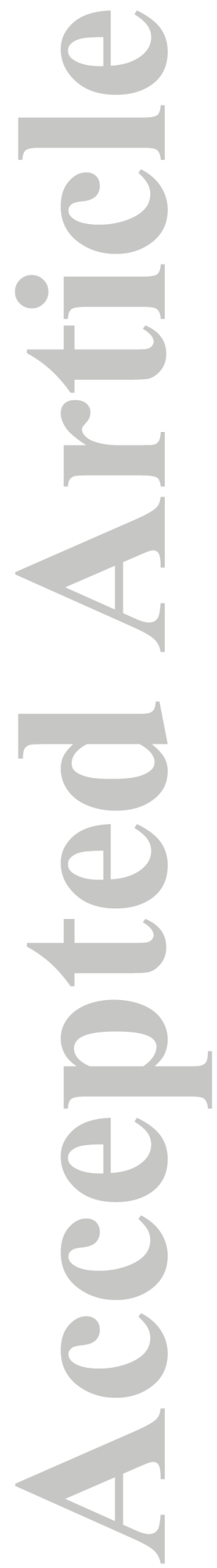

This article is protected by copyright. All rights reserved. 